\title{
Development of a Smoothed Particle Hydrodynamics Method and its Application to Aircraft Ditching Simulations
}

\author{
Tianhang Xiao ${ }^{* 1}$, Ning Qin ${ }^{\dagger 2}$, Zhaoyan Lu $^{\ddagger 1}$, Xuan Sun ${ }^{\S 1}$, Mingbo Tong ${ }^{* * 1}$, Zhengzhong Wang ${ }^{\dagger 3}$ \\ 1. Nanjing University of Aeronautics and Astronautics, Nanjing, 210016, P.R. China \\ 2. The University of Sheffield, Sheffield, S1 3JD, UK \\ 3. Helicopter Research and Development Institute, Jingdezhen, 333001, P. R. China
}

\begin{abstract}
The present study addresses the development and validation of a smoothed particle hydrodynamics (SPH) method, particularly to examine its feasibility and capability in hydrodynamics and dynamics of aircraft during ditching. The developed method solves the weakly compressible Navier-Stokes equations coupled with six-degree of freedom dynamics to achieve an accurate prediction of the interaction between the aircraft and the fluid. In this SPH method, a dummy particle wall-boundary condition is automatically implemented to meet the requirement of application on geometrically complex engineering problems. An efficient particle search strategy merging the ideal of Cell-linked list with Vertlet list is proposed to speed up the neighbor particles search process. The present SPH method uses an OpenMP memory-shared parallelization in conjunction with Z-curve reordering to accelerate the computation. Validations have been performed on several classic hydrodynamic problems, where good agreements were achieved via comparing with documented experimental results. The developed SPH method is applied to predict the ditching event of a complex helicopter model. Results demonstrate the ditching process, indicating that the method can be potentially used in aircraft ditching applications.
\end{abstract}

Keywords: smoothed particle hydrodynamics; aircraft ditching; water entry; particle neighbor search; dummy particle boundary condition; weakly compressible SPH

\footnotetext{
${ }^{*}$ Associate Professor, College of Aerospace Engineering, 29 Yudao Street; Visiting Scholar, Department of Mechanical Engineering, University of Sheffield. xthang@ nuaa.edu.cn

${ }^{\dagger}$ Professor of Aerodynamics, Department of Mechanical Engineering, Mappin Street.

${ }^{*} \mathrm{PhD}$ student, College of Aerospace Engineering, 29 Yudao Street.

${ }^{\S}$ PhD student, College of Aerospace Engineering, 29 Yudao Street.

** Professor, College of Aerospace Engineering, 29 Yudao Street.

${ }^{\dagger}$ Professor, Science and Technology on Rotorcraft Aeromechanics Laboratory, 16 Hangkong Road.
} 


\section{Introduction}

DITCHING is one of the most extreme emergency circumstances that ends with an intentional impact of the aircraft with water. Aircraft ditching on water is a very complex physical problem, which involves a wide range of disciplines such as kinematics, stability, hydrodynamics and structural engineering, thus makes the analysis of such problems a multi-disciplinary task. Additionally, the dynamic responses applied to the structure during an impact on water are significantly different from those happening during an impact on a rigid ground. The aforementioned facts

eventually lead to the necessity of performing experimental and numerical investigations in order to improve the survivability and structure tolerance when aircraft ditching occurs.

Experimental model drop test particularly using the full-scale aircraft $[1,2]$ is regarded as one of the most straightforward ways to predict the ditching events. However, due to the economical and practical reasons, scaledmodel test with model launched in a water tank is more feasible especially when various ditching scenarios are considered. An example is the ditching test performed by Climent et al. [3] for the sake of investigating the ditching behavior of a military aircraft $\mathrm{CN}-235-300 \mathrm{M}$ where a $1 / 8$ scaled model was used. More recently, an extensive test campaign of guided ditching has been conducted by CNR-INSEAN [4] within the working package of the FP7 research project SMAES aiming to provide available experimental database for validation. However, experimental tests are generally expensive, and thus only a narrow number of geometries can be tested during the aircraft design stage. There are also other limitations related to experimental testing, such as repeatability issues arose from the unensured precise ditching motion and the inherent but undesirable scale effects in hydrodynamics.

Numerical ditching analysis ranges from simple analytical method to numerical simulation methods. A variety of analytical /semi-analytical methods are documented to simulate water impact, e.g., von Karman's estimation method based on momentum theory [5, 6], the simple analytical method introduced by Farhad [7] derived from the linear potential flow theory and the very recent semi-analytical method for a plate impacting on water problem [8]. In view of the fact that for highly non-linear hydrodynamic behavior of complex geometry with potential failure of realistic structures, analytical /semi-analytical approaches are inappropriate to solve this fluid-structure interaction problem. Numerical methods provide alternative solutions to predict the effects of aircraft ditching due to their abilities in solving this type of non-linear hydrodynamics.

During the past decades, the mesh-based Lagrangian methods, e.g. Finite Element Method (FEM), were widely applied to water impact. Vignjevic [9], Pentecote and Vigliotti [2], and Ortiz et al. [10] used the FEM to numerically 
simulate the vertical impact on water of the conventional helicopter structure for crashworthiness assessment purposes. However, the violent nature of the considered aircraft ditching problems causes the mesh-based Lagrangian methods to fail in accurately describing the fluid flow because they cannot handle significantly large mesh distortion. Arbitrary Lagrangian-Eulerian (ALE) methods were later considered as an alternative way to overcome the limitation of Lagrangian FEM due to its inherent continuous rezoning capability. With the freedom in moving the computational mesh offered by the ALE description, greater distortions of the continuum can be handled than that would be allowed by a purely Lagrangian method. This also provides more resolution than that offered by a purely Eulerian scheme. Jackson and Fuchs [11] simulated the water impact using ALE approach in the LS-DYNA software, which is a non-linear, explicit transient dynamic finite element model. Their results showed that the ALE method can provide reasonable pre-test predictions of the floor level acceleration responses. Hua et al.[12] proposed a three-dimensional dynamic structural model with the real geometry of an aircraft and an ALE fluid field model to simulate the fluid-solid interactions caused by low speed ditching using the LS-DYNA with experimental validation. Similar ALE model has been created by Hu et al. [13] and further applied to a full-scaled shape of Boeing 777-200 model. However, as a mesh-based method, the ALE approach relies heavily on high quality mesh and may inherently suffer from the poor mesh caused by large deformation and complex free surface geometries. Moreover, the ALE methods face some other potential problems, such as accuracy in free surface tracking, severe fluctuation of pressure field due to variation of mesh density and leakage modeling of surface-to-surface interaction.

Another mesh-based approach applied to water ditching of aircraft is the Finite Volume Method (FVM) coupled with free surface tracking method, e.g. the Volume of Fluid (VOF) method, where the aerodynamic and hydrodynamic forces can be derived. Wick [14] performed simulations to study the splashdown of an unmanned air vehicle (UAV) taking nose dive into seawater from various heights with a range of impact velocities. They used a time-accurate FVM based on the unsteady compressible ensemble averaged Navier-Stokes equations for the air and the unsteady incompressible ensemble averaged Navier-Stokes equations for the seawater. Guo et al. [15] used the Unsteady Reynolds-averaged Navier-Stokes (URANS) equations to numerically investigate the effect of pitch angle on the impact characteristics of water ditching. The transformation of the air-water interface is treated by the VOF model. Further study carried out by Qu et al. [16] imposed the global-moving-mesh method to deal with the relative motion between the water and the object. Results illustrated that the global-moving-mesh method can avoid the high computation expense and low-quality mesh in the conventional ALE mesh-deforming methods. One of the big 
issues of FVM for water impact problems is that a free surface tracking method is additionally needed and the accuracy of FVM is mostly dependent on the accuracy of free surface tracking. Similar to the mesh-based Lagrangian or ALE methods, the FVM method also suffers from the aspect of dynamic mesh deformation which not only increases additional computational cost but torments or even aborts computation if the mesh is not treated very carefully.

The meshless Lagrangian Smoothed Particle Hydrodynamics (SPH) method [17] is identified as well-suited for solving water impact problems by the violent nature of a ditching event which involves high deformation, nonlinear phenomena and complex free surface shapes. The SPH method divides the continuous medium into a set of particles and integrates the fluid governing equations on each particle in Lagrangian formula. The physical quantity of any particle is computed by an interpolation of the values of the nearest neighbor particles, and then particles move according to these quantities. For fluid flow, the pressure field is obtained by a weakly compressible state equation [18] or by additionally solving a pressure Poisson equation [19,20,21]. Without a mesh, the SPH method makes itself more convenient to describe the violent fluid deformation and could principally avoid the problem with distortion of the mesh. In addition, the Lagrangian frame will leave out the spatial discretization of the convection term in the governing equations, preventing the consequent diffusion. Recently, a few softwares or open-source SPH codes, e.g. SPHysics [22], are being developed and has been successfully applied on environmental, oceanic and coastal engineering problems $[23,24,25]$. Besides, the SPH method was also extended to be a potential useful tool in the aeronautical applications. Groenenboom et al. [25,26] implemented the SPH method into an explicit finite element program and one of their applications is to analysis ditching and floating behavior of a helicopter with external flotation system. The results demonstrated the capabilities of the SPH method to solve complex aircraft ditching problems. Later, within the European SMAES project, efforts have been made by Groenenboom [27,28], Benítez et al.[29], to extend the SPH module within the hybrid FE-SPH code VSP with various innovative feathers. Further works $[30,31,32]$ that attempted to couple the SPH with the structural finite element model provided new possibilities to investigate the structure-fluid interactions with structure deformation taken into consideration during an aircraft ditching event.

The main work of this paper is to develop a SPH-based numerical method in order to investigate the specific hydrodynamics and dynamics of aircrafts during ditching events. The numerical framework of the in-house developed SPH code is introduced in Section 2, where a weakly compressible SPH method coupled with six-degree- 
freedom dynamics is presented. Also, an automatic dummy particle wall boundary treatment is developed for threedimensional complex geometries to meet the requirements of engineering applications. Section 3 subsequently presents the solution strategies of SPH, high efficiency particles searching method and parallel computing issue, in which, the implementation strategies of efficient neighbor search and memory-shared OpenMP parallelization in conjunction with Z-curve reordering are highlighted. To assess the capabilities of the SPH method, several engineering applications are validated in terms of numerical accuracy and computational efficiency in Section 4.

\section{SPH Methods}

\subsection{Governing Equations}

The governing equations for the motion of an iso-thermal fluid in a Lagrangian frame are the continuity equation,

$$
\frac{\mathrm{d} \rho}{\mathrm{d} t}=-\rho \vec{\nabla} \cdot \vec{v}
$$

and the momentum equation,

$$
\frac{\mathrm{d} \vec{v}}{\mathrm{~d} t}=\frac{1}{\rho} \vec{\nabla} \underset{\underline{\sigma}}{\underline{S}}+\vec{S}
$$

where $\rho$ and $\vec{v}$ are the density and velocity vector of the fluid, respectively. $\vec{S}$ is the body force which for the problems in the present study is gravity. The stress tensor of the element is defined as,

$$
\underline{\underline{\sigma}}=-p \underline{\underline{I}}+\underline{\underline{\tau}}
$$

For a Newton fluid, the stress tensor can be derived as a linear equation formulated as

$$
\underline{\underline{\sigma}}=-\left(p+\mu^{\prime} \vec{\nabla} \cdot \vec{v}\right) \underline{\underline{I}}+2 \mu \underline{\underline{D}}, \quad \mu^{\prime}=-\frac{2}{3} \mu .
$$

To close the equations, the fluid is treated as weakly compressible [18]; in this way it is easier to determine the fluid pressure by using an equation of state, which is much faster than solving a differential equation like the Poisson's equation used in the incompressible method. The relationship between pressure and density follows the Tait's equation of state and can be written as,

$$
p=B\left[\left(\frac{\rho}{\rho_{0}}\right)^{\gamma}-1\right]
$$

where $\rho_{0}$ is the reference density defined as the initial density at the free surface, and $\gamma$ is the polytrophic constant,

normally $\gamma=7$ for water. The choice of $B$, which is related to the fluid compressibility modulus and determines the 
speed of sound at the reference density, $B=\rho_{0} c_{0}^{2} / \gamma$, should be selected to restrict the sound speed to be at least ten times faster than the maximum fluid velocity, thereby keep density variations within $1 \%$.

\subsection{Mathematical Concept of SPH}

The SPH formulation is based on an integral interpolation. A generic function $A(\vec{r})$ of the position vector $\vec{r}$ can be represented as

$$
A(\vec{r})=\int A\left(\vec{r}^{\prime}\right) \delta\left(\vec{r}-\vec{r}^{\prime}\right) \mathrm{d} \vec{r}^{\prime}
$$

where $\delta\left(\vec{r}-\vec{r}^{\prime}\right)$ is Dirac $\delta$-function. Since the Dirac $\delta$-function lacks some required properties for a "well behaved function" such as continuity and differentiability, it is replaced by a kernel function, and $A(\vec{r})$ is approximated as,

$$
\langle A(\vec{r})\rangle=\int A\left(\vec{r}^{\prime}\right) \mathrm{W}\left(\vec{r}-\vec{r}^{\prime}, h\right) \mathrm{d} \vec{r}^{\prime},
$$

where $\mathrm{W}$ is the kernel or weighting function; $h$ is the smoothing length defining the interaction distance and controlling the size of the integral domain. The above, in discrete notation, leads to the an approximation of the function and its derivative at a particle $i$ as,

$$
A\left(\vec{r}_{i}\right)=\sum_{j} m_{j} \frac{A_{j}}{\rho_{j}} \mathrm{~W}_{i j}, \quad \vec{\nabla} A\left(\vec{r}_{i}\right)=\sum_{j} m_{j} \frac{A_{j}}{\rho_{j}} \vec{\nabla}_{i} \mathrm{~W}_{i j}
$$

where $m_{j}, \rho_{j}$ denotes the mass and density of particle $j$ respectively. $\mathrm{W}_{i j}=\mathrm{W}\left(\vec{r}_{i}-\vec{r}_{j}, h\right)$ is the kernel function and $\vec{\nabla}_{i} \mathrm{~W}_{i j}$ is its derivative which can be analytically calculated. The summation is over all the particles $j$ within the region of compact support of the kernel function.

The selected kernel function should fulfill the mathematical constraints of positivity, compact support, normalization and Delta function behavior. There are a wide variety of kernel functions available and the cubicspline kernel function is adopted in the present study as it is probably one of the most commonly used kernels because of its simplicity and cheap computational cost. The cubic-spline kernel can be written as,

$$
\mathrm{W}\left(r_{i j}, h\right)=\alpha_{D}\left\{\begin{array}{lc}
\frac{2}{3}-q^{2}+\frac{1}{2} q^{3} & 0 \leq q<1 \\
\frac{1}{6}(2-q)^{3} & 1 \leq q \leq 2 \\
0 & q>2
\end{array}\right.
$$


where $r_{i j}=\left|\vec{r}_{i}-\vec{r}_{j}\right|$ is the Euclidean distance between particle $i$ and $j, h$ is the smoothing length, and $q=\frac{r_{i j}}{h} . \alpha_{D}$ is $15 /\left(7 \pi h^{2}\right)$ for $2 \mathrm{D}$ case and $3 /\left(2 \pi h^{3}\right)$ for $3 \mathrm{D}$ case.

\subsection{SPH Formulation}

The discretized form of the continuity equation Eqn. (1) for particle $i$ is

$$
\frac{\mathrm{d} \rho_{i}}{\mathrm{~d} t}=\sum_{j=1}^{N} m_{j} \vec{v}_{i j} \cdot \vec{\nabla}_{i} W_{i j}
$$

where $m_{j}$ is the mass the particle $j, \vec{v}_{i j}=\vec{v}_{i}-\vec{v}_{j}$ is the relative velocity vector between particle $i$ and $j$.

The momentum equation can be formulated in SPH notation as [33]

$$
\frac{\mathrm{d} \vec{v}_{i}}{\mathrm{~d} t}=-\sum_{j=1}^{N} m_{j}\left(\frac{p_{i}}{\rho_{i}^{2}}+\frac{p_{j}}{\rho_{j}^{2}}\right) \vec{\nabla}_{i} W_{i j}+\text { viscous terms }+\vec{g},
$$

where $\rho_{j}$ and $p_{j}$ are density and pressure of particle $j$, and $\vec{g}$ is the gravitational acceleration.

To calculate the position of the particles, the XSPH [18] version, instead of the standard SPH method, was employed in this study for reducing the disorder issue. The position of the particles are calculated with a smoothed velocity as,

$$
\frac{\mathrm{d} \vec{r}_{i}}{\mathrm{~d} t}=\vec{v}_{i}-\varepsilon \sum_{j=1}^{N} \frac{m_{j}}{\bar{\rho}_{i j}} \vec{v}_{i j} W_{i j}
$$

where $\varepsilon$ is the relaxation factor, $0 \leq \varepsilon \leq 1.0$, typically chosen at 0.5 . The XSPH moves each particle with a smoothed velocity which is derived from averaging the velocities of its neighbors, therefore, the position fluctuations are reduced significantly.

It should be mentioned that some strategies are applied to the above SPH formulation to improve its accuracy and stability, e.g., the tensile instability problem is suppressed by adding an artificial stress term [34,35] in the momentum equation which introduces a small short-range repulsive force between a pair of neighboring particles to prevent them from getting too close; a periodical re-initialization of density field by using the Shepard filter is applied every 20-30 time steps to illuminate the large pressure oscillation problems caused by the acoustic waves presented in compressible fluids.

The viscous terms in Eqn. (11) are treated in two ways: artificial viscosity and laminar model.

\section{Artificial viscosity}

Applying the artificial viscosity [18] in the SPH notation, the momentum equation can be formulated as, 


$$
\frac{\mathrm{d} \vec{v}_{i}}{\mathrm{~d} t}=-\sum_{j=1}^{N} m_{j}\left(\frac{p_{i}}{\rho_{i}^{2}}+\frac{p_{j}}{\rho_{j}^{2}}+\Pi_{i j}\right) \vec{\nabla}_{i} W_{i j}+\vec{g},
$$

where the artificial viscosity item $\Pi_{i j}$ will bring in a repulsion force when two particles approach each other. The general formation is,

$$
\Pi_{i j}=\left\{\begin{array}{cc}
-\frac{\alpha \mu_{i j} \bar{c}_{i j}}{\bar{\rho}_{i j}} & \vec{v}_{i j} \cdot \vec{n}_{i j}<0 \\
0 & \vec{v}_{i j} \cdot \vec{n}_{i j} \geq 0
\end{array}, \mu_{i j}=h \frac{\vec{v}_{i j} \cdot \vec{r}_{i j}}{\left|\vec{r}_{i j}\right|^{2}+\eta^{2}}\right.
$$

where $\alpha$ is a user-defined parameter to describe the strength of viscosity; $\bar{\rho}_{i j}, \bar{c}_{i j}$ are the mean density and sound speed of particle $i, j ; \vec{n}_{i j}=\vec{r}_{i j} /\left|\vec{r}_{i j}\right| ; \vec{v}_{i j}=\vec{v}_{i}-\vec{v}_{j} ; \eta^{2}=0.01 h^{2}$. When the above artificial viscosity is used in the absence of a physical viscosity, an equivalent effective physical kinematic viscosity $v$ can be computed from $\alpha$ as $v=\frac{\alpha h \bar{c}_{i j}}{2(d+2)}$, where $d$ is the number of dimensions.

\section{Laminar model}

If a laminar model is applied on the SPH method, the momentum equations of Eqn. (2) can be written as,

$$
\frac{\mathrm{d} \vec{v}_{i}}{\mathrm{~d} t}=-\frac{1}{\rho} \nabla p+\vec{g}+v_{0} \nabla^{2} v
$$

and the laminar viscous term is formulated in SPH notation as,

$$
\left(v_{0} \nabla^{2} v\right)_{i}=\sum_{j=1}^{N} m_{j} \frac{4 v_{0} \vec{r}_{i j} \cdot \vec{\nabla}_{i} W_{i j}}{\left(\rho_{i}+\rho_{j}\right)\left(\left|\vec{r}_{i j}\right|^{2}+\eta^{2}\right)} \vec{v}_{i j} .
$$

\subsection{Solid Wall Boundary Condition}

For the SPH method, the treatment of wall boundary condition is challenging due to the fact that the support domain of the kernel function is cut off by the domain boundary as particles approach a rigid boundary where the compact support condition of kernel function cannot be satisfied. Therefore, special treatment must be imposed on the boundaries. Following the work of Adami et al. [36], the present study uses the so called dummy particles to model solid wall boundaries to ensure that the support of the kernel interpolations is fully contained within the fluid phase for density change and force calculation (see Figure 1). The pressure at a dummy particle position is computed from its surrounding fluid particles based on a local force balance between wall and fluid particles, which can be used to prevent wall penetration of the fluid particles. Both slip or non-slip wall boundary conditions can be 
imposed for either stationary or moving walls by assigning the wall particle with an approximate moving velocity. In the present study, dummy particles along with fluid particles are automatically generated and initialized when the geometries and the necessary information of computation are imported. For complex CAD geometries (Figure 2(a)), the surface mesh is generated firstly according to the size of particle (Figure 2(b)), and then two or three layers of dummy particle are generated perpendicularly to the surface (Figure 2(c)); while for simple geometries, such as pools or tanks, the particles are generated analytically with respect to the user inputs.

This dummy particle method, compared to other wall boundary treatments, e.g., mirror particle and contact algorithms/impulsive force methods, was proved to be as a simple way for complex geometries. This method gives a clear description of the geometric boundaries throughout the simulation once the particles have been initialized. Unlike the contact algorithm where an additional penalty separation stress [29] has to be employed to mimic suction effect, this method can model such effect accurately without any additional treatment. Since the dummy particles reflect both the physical properties of fluid around them and the motion dynamics of solid walls, the interactions of the dummy and fluid particles inherently mimic the hydrodynamic effects caused by the interactions between the solid wall and the fluid. Overpressure occurs in regions where the velocity of fluid is low, and dummy and fluid particles are approaching each other. Suction forces arise in some areas, e.g., near the convex-curved region at the rear of the fuselage, where fluid particles accelerate under the sticky contact of dummy particles, leading to a drop of fluid pressure when fluid particles detaching from the dummy particles.

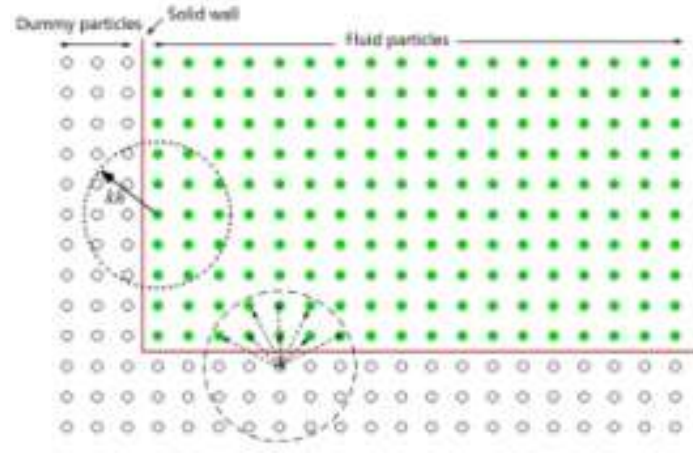

(a)

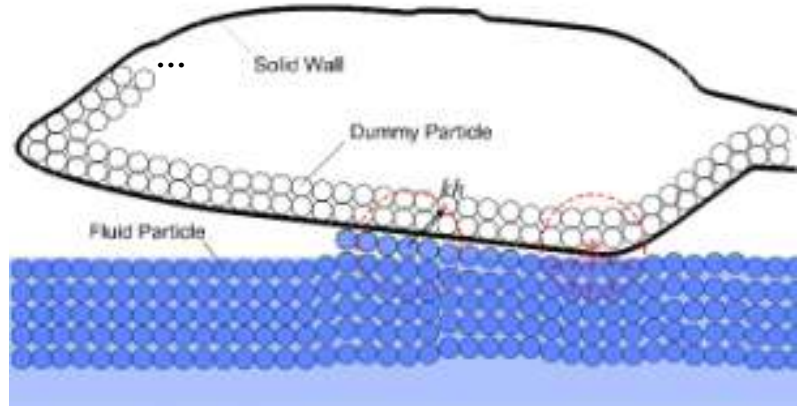

(b)

Figure 1 Schematic illustration of the wall boundary condition ((a) static pool wall; (b) ditching object): (•) represents the fluid particles and $(0)$ is the dummy particles. 


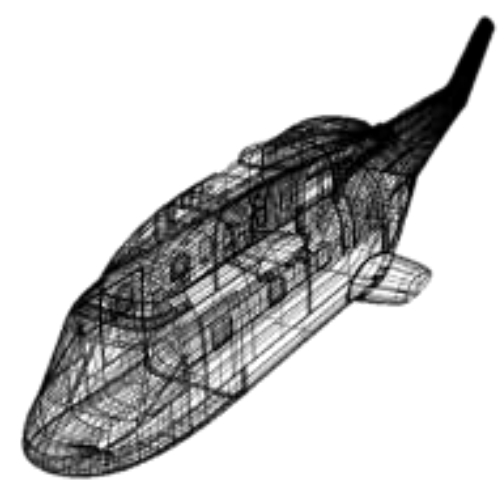

(a)

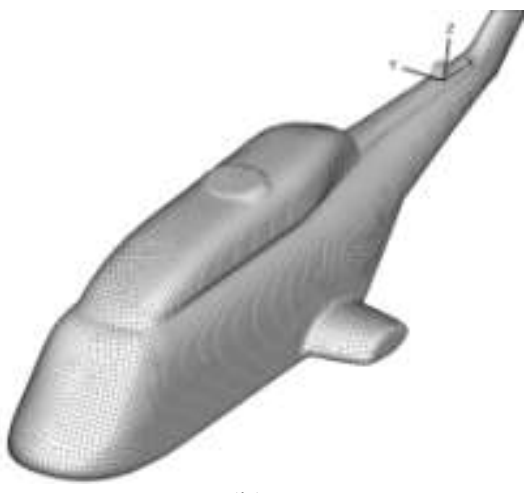

(b)

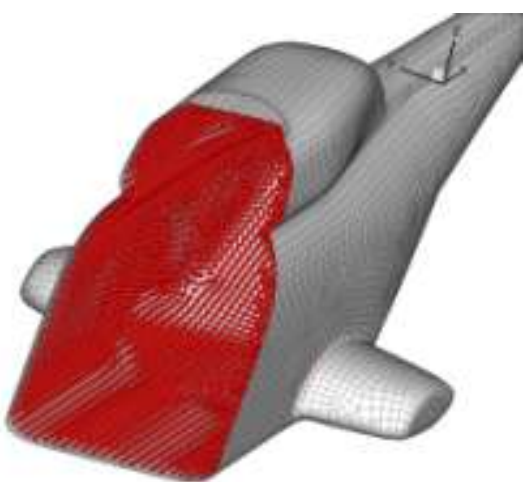

(c)

Figure 2 Example of dummy particles generation for a complex helicopter model:(a) CAD model; (b) surface mesh; (c) cut-plane view of the dummy particles (solid red dots) generated perpendicularly to the surface.

\subsection{Time step algorithm}

The density, momentum and position equations can be formulated in one similar differential equation,

$$
\frac{\mathrm{d} \mathbf{U}_{i}}{\mathrm{~d} t}=\mathbf{R H S}_{i}
$$

where

$$
\mathbf{U}=\left(\begin{array}{c}
\rho \\
\vec{v} \\
\vec{x}
\end{array}\right), \quad \mathbf{R H S}=\left(\begin{array}{c}
\sum_{j=1}^{N} m_{j} \vec{v}_{i j} \cdot \vec{\nabla}_{i} W_{i j} \\
-\sum_{j=1}^{N} m_{j}\left(\frac{p_{i}}{\rho_{i}^{2}}+\frac{p_{j}}{\rho_{j}^{2}}\right) \vec{\nabla}_{i} W_{i j}+\text { viscous term }+\vec{g} \\
\vec{v}_{i}-\varepsilon \sum_{j=1}^{N} \frac{m_{j}}{\bar{\rho}_{i j}} v_{i j} W_{i j}
\end{array}\right) .
$$

The above equations can be integrated in time with the predictor-corrector method [37]. A multi-stage RungeKutta scheme is also implemented here so that more options are available. For easy coding, these two integration algorithms are written in a unified form as follows:

$$
\begin{aligned}
& \mathbf{U}_{i}^{n(m)}=\mathbf{U}_{i}^{n}+\Delta t \sum_{k=1}^{m-1} a_{m k} \mathbf{R H S}_{i}\left(\mathbf{U}_{i}^{n(k)}\right) ; \quad 1 \leq m \leq M \\
& \mathbf{U}_{i}^{n+1}=\mathbf{U}_{i}^{n}+\Delta t \sum_{k=1}^{M} b_{k} \mathbf{R H S}_{i}\left(\mathbf{U}_{i}^{n(k)}\right)
\end{aligned}
$$

where $M$ is the maximum inner stages and the constant coefficients $a_{m k}, b_{k}(1 \leq m, k \leq M)$ listed in Table $\mathbf{1}$ can be used to define the method and accuracy of the time integration scheme. Note that only the predictor-corrector, 2stage and 4-stage Runge-Kutta algorithms are used and compared in the present study, but the coefficients of 3-stage 
Runge-Kutta are also presented in Table 1 for completeness. During the time integration computation, a variable time step size $\Delta t$ is calculated following the work of Monaghan and Kos [38] where the Courant-Friedrich-Levy (CFL) condition, the force terms and the viscous diffusion term are considered to keep the algorithm stable.

Table 1 Coefficients of the temporal integration scheme

\begin{tabular}{c|c|ccccc|cccc}
\hline \hline \multicolumn{1}{c}{ Algorithm } & \multicolumn{1}{c}{$\alpha_{k}\left(a_{m k}\right)$} & \multicolumn{5}{c}{$\beta_{k}\left(b_{k}\right)$} \\
\hline Predictor-Corrector & 2 & 0.5 & 1 & $/$ & $/$ & 0 & 1 & $/$ & $/$ \\
2-stage Runge-Kutta & 2 & 1 & 1 & $/$ & $/$ & 0.5 & 0.5 & $/$ & $/$ \\
3-stage Runge-Kutta & 3 & $1 / 3$ & $2 / 3$ & 1 & $/$ & $1 / 4$ & 0 & $3 / 4$ & $/$ \\
4-stage Runge-Kutta & 4 & 0.5 & 0.5 & 1 & 1 & $1 / 6$ & $1 / 3$ & $1 / 3$ & $1 / 6$ \\
\hline \hline
\end{tabular}

\subsection{Six-Degree of Freedom Equations of motion}

For aircraft ditching simulation, the motion of the aircraft is determined by a six-degree of freedom (6DOF) calculation so that the interaction between the aircraft and the fluid can be accurately captured. The SPH computation determines the fluidic forces and moments that act on the body of aircraft. In turn, these forces and moments, together with other forces and moments i.e. gravitational force, are used in the general equations of motion to calculate the translational and rotational displacement of the aircraft body. The governing equation for the translational motion of the center of gravity in the inertial coordinate system is given as,

$$
m \dot{\vec{v}}_{\mathrm{G}}=\sum \vec{F}+m \vec{g}
$$

where $m$ is the mass of the body, $\vec{v}_{\mathrm{G}}$ is the translational motion of the center of gravity and $\vec{F}$ is the external force vectors acting on the body; while the angular motion of the moving body is computed in the body coordinates to avoid time-variation inertia properties,

$$
\mathbf{I} \dot{\vec{\omega}}_{\mathrm{B}}=\sum \vec{M}_{\mathrm{B}}-\vec{\omega}_{\mathrm{B}} \times \mathbf{I} \vec{\omega}_{\mathrm{B}}
$$

where $\mathbf{I}$ is the inertia tensor, $\vec{\omega}_{\mathrm{B}}$ is the angular motion in body coordinate and $\vec{M}_{\mathrm{B}}$ is the moment vector.

The above two equations of motion are solved by a 4-stage Runge-Kutta time integration method in the present study.

\section{Implementation Strategies}

\subsection{Neighbor Particles Search}

Neighbor particle search (required when discretizing gradient) of the SPH method could be time-consuming 
due to the fact that grid connectivity information is not available, such circumstance requires an efficient algorithm to search neighbor particles. There are two conventional methods were used to accelerate the generation of neighbor particle list [39,40], namely Cell-linked list and Verlet list. Dominguez [40] compared both methods and indicated that both approaches have the similar searching ability in terms of computational time for each construction of neighbor list while the Verlet list requires more memory storage. The present study proposed a search strategy by merging the idea of Cell-linked list with the Verlet list algorithm that not only maintains the efficiency of Cell-linked list but also takes advantage of the possibility of keeping the same list during several consecutive time steps to reduce the total computational cost. The search scheme in the present study can be summarized into two steps: (1) creation of background grid cells for particle registration and (2) construction of list of potential particle pairs for force computation. The detail algorithms of the aforementioned steps are:

(1) Create background grid cells and register the particles to the cell they belong to.

- Loop all the particles (both fluid and dummy particles) to find out the maximal and minimal spatial coordinates, i.e., $\left(x_{\mathrm{Max}}, y_{\mathrm{Max}}, z_{\mathrm{Max}}\right),\left(x_{\mathrm{Min}}, y_{\mathrm{Min},} z_{\mathrm{Min}}\right)$ and the smooth length $h_{\max }$.

- Divide the computational domain $\left[\left(x_{\mathrm{Min}}, y_{\mathrm{Min}}, z_{\mathrm{Min}}\right),\left(x_{\mathrm{Max}}, y_{\mathrm{Max}}, z_{\mathrm{Max}}\right)\right]$ into cells of side $\mathrm{d} s=2 h_{\max }+\varepsilon$ ( where $\varepsilon$

is the tolerance of the Verlet-list and will be discussed later ) and assign each cell with an index of Cell_Index $(i, j, k)$ by the way similar to structural grid as,

$$
\begin{aligned}
& \text { Cell_Index }(i, j, k)=i+j \cdot \operatorname{IMAX}+k \cdot \mathrm{JMAX} \cdot \mathrm{IMAX}, \text { where } \\
& \mathrm{IMax}=\operatorname{int}\left[\left(x_{\mathrm{Max}}-x_{\mathrm{Min}}\right) / \mathrm{d} s\right]+1 \\
& \mathrm{JMax}=\operatorname{int}\left[\left(y_{\mathrm{Max}}-y_{\mathrm{Min}}\right) / \mathrm{d} s\right]+1 \\
& \mathrm{KMax}=\operatorname{int}\left[\left(z_{\mathrm{Max}}-z_{\mathrm{Min}}\right) / \mathrm{d} s\right]+1
\end{aligned}
$$

- Calculate the Cell_Index of each particle by its position and register particles (store the address of particles) to the cell they belong to according to the Cell_Index $(i, j, k)$ with,

$$
\left.\left.\left.i=\operatorname{int}\left[\left(x-x_{\text {Min }}\right) / \mathrm{d} s\right)\right], \quad j=\operatorname{int}\left[\left(y-y_{\text {Min }}\right) / \mathrm{d} s\right)\right], \quad k=\operatorname{int}\left[\left(z-z_{\text {Min }}\right) / \mathrm{d} s\right)\right] .
$$

(2) Search neighbor particles and construct list for potential particle pairs.

- Loop all the fluid particles following the order of particle number. For each fluid particle, i.e. $a$, search its potential neighbors in the adjacent cells. When the distance between particle $a$ and another particle $b$ (fluid particle with number larger than $a$ or dummy particle) is less than $\mathrm{d} s$, make a pair of them, $\operatorname{pair}(a, b)$ and add the particle pair to the list. 
Note that, after constructed, the particle pair list contains not only the pairs of particles with distance less than $2 h$ which do interact at current time step, but the candidate pairs of particles which may interact during the next following time steps. As particles move in time, the interacting particle pairs can change at each time step. The particle pair list is loaded at each time step for force computation and keeps unchanged during the following consecutive steps until that a particle leaves or enters the neighborhood of the particle and thus the list should be refreshed.

It is obvious that the value of $\varepsilon$ determines the list length and the refreshing frequency. If $\varepsilon=0$, the standard Celllinked list approach is recovered, and the list will be refreshed at every each time step. However, if $\varepsilon$ is chosen too large, the pair list could be undesirable longer, which implies higher memory requirement and will slow down the solution since the number of false candidate pairs increases.

To reach a balance of list length and refresh frequency, the value $\varepsilon=\max \left(0.05 h, \min \left(0.2 h, 2 n\left|V_{\max }\right| \Delta t\right)\right)$ is proposed with $n$, the number of steps that the list is desirably kept. Meanwhile, checking should be conducted at each time step to see if the list refreshing condition is established since the value of $\varepsilon$ is tentative while the particles dynamically move in time. Following the work of Dominguze [40], the position of particles in the domain are recorded at the time step when the pair list constructed. During the following time steps, the position of particles is checked. When the distance travelled by any particle from the recorded time step is larger than $\varepsilon / 2$, the list is reconstructed and assumed to last for next $n$ steps. It should be noticed that the pair list can be kept for more than $n$ time steps when the particles do not move dramatically, i.e., at the beginning stage of dam break; or the list may be reconstructed for the frequency less than $n$ steps due to the violent particle motion. This method was tested to be ideal to maintain the pair list at a reasonable length whilst to keep the list unchanged for a few steps even at the most violent event.

\subsection{Memory-Shared Parallelization and Particle Reordering}

In the present study, an OpenMP-based memory shared parallelization strategy is developed to accelerate the SPH computation due to the fact that a shared memory space suggests easier parallelization. However, there are two issues, i.e., data races and cache misses, which should be considered when memory shared parallelization is applied.

The interacting force inside a particle pair is calculated and contributes to each particle reciprocally, exploiting the symmetry of the interaction. There exists a scenario that one particle is involved in several particle interacting pairs, so the parallelization of force computation is not straightforward due to data races. As the particle pairs are 
constructed explicitly in the present study, the most convenient way to avoid memory contention is to group the particle pairs ensuring that none of the corresponding particles can be accessed more than once within each group of particle pairs. In such a way, the force computation can be processed in parallel for each group of particle pairs without race condition. A simply coloring algorithm proposed by Lonhner [41] is employed to group the particle pairs in the present study.

During the SPH computation, it is important to take advantage of spatial and temporal data locality to allow an efficient use of caches and a reduction of data transfer. However, for the water impact problems in this paper, particles and the interaction with their neighbors dynamically vary both in space and in time with data locality broken frequently, and thus efficient particle reordering algorithm is required to keep the interacting particles in the vicinity in memory. The present study suggests to regularly reorder the particles according to a space filling curve, i.e. Z-curve, to preserve data locality.

At each time of reordering, the integral coordinate of each particle, $I C(i, j, k)$ is firstly calculated from its position by a similar way for Cell_Index in Section 3.1 with

$$
\left.\left.\left.i=\operatorname{int}\left[10 \times\left(x-x_{\text {Min }}\right) / \mathrm{d} s\right)\right], j=\operatorname{int}\left[10 \times\left(y-y_{\text {Min }}\right) / \mathrm{d} s\right)\right], k=\operatorname{int}\left[10 \times\left(z-z_{\text {Min }}\right) / \mathrm{d} s\right)\right],
$$

where the coefficient of ten is used and is large enough to guarantee a unique integral coordinate for each particle, and then the index, i.e. Z-value, can be simply computed by interleaving the binary representations of its integral coordinate values. According to the Z-values, particles can be sorted into an order of "Z-curve" recursive style as illustrated in Figure 3. As can be seen, spatial locality is improved significantly with respect to the original order (Figure 3(a)), which thanks to the self-containing block structure of the Z-curve ordering, and consequently, spatial compactness enforced by this ordering would lead to a high cache-hit.

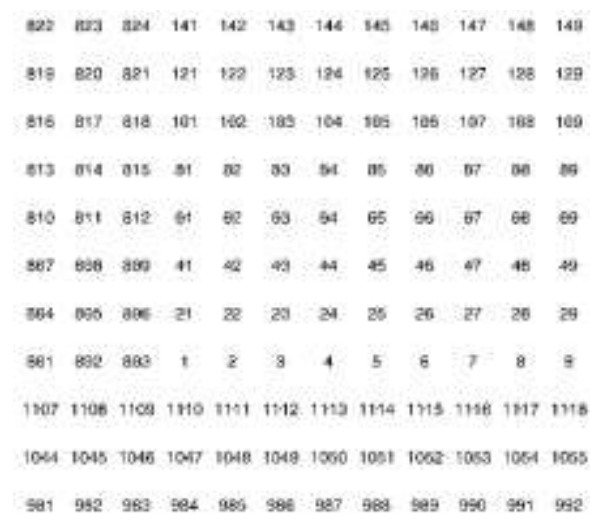

(a)

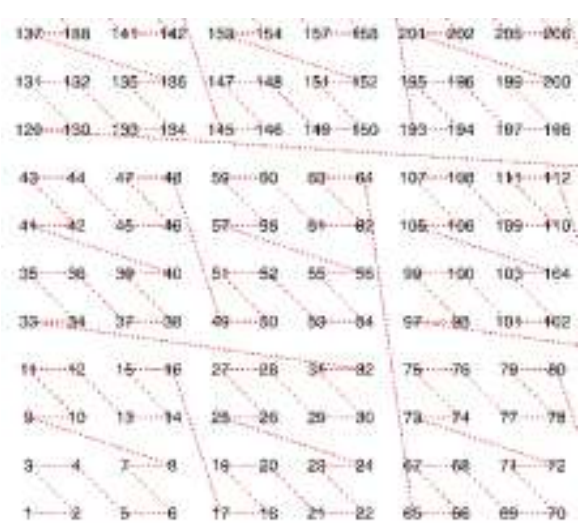

(b)

Figure 3 Illustration of Z-curve reordering of particles: (a) particle order before reordering; (b) particle order after $\mathrm{Z}$-curve reordering. 
The particles carry a large amount of physical information, e.g. position, density, velocity, pressure and other auxiliary computational data, and these data also have to be translated when sorting the particles. The memory transfer would thus slow down the sorting process significantly if the particle array itself is sorted at each time step. Instead of directly sorting the particle array itself, a secondary data structure is employed which stores a key-value pair referring to a particle (value) and its corresponding Z-value (key). Because of the minimal memory usage of the pairs, sorting the pairs is much more efficient than sorting the particle array itself. To enforce spatial compactness, the particle array itself will still be reordered. Since particle cannot move more than half of the smooth length in one step to satisfy the CFL condition and thus the fluid particles undergo slow and coherent movements in one step, the average percentage of particles with order changed in a consecutive step is very small once the particles are initially reordered at the first step. So, in the present study, only the particles with their orders changed are adjusted in memory according to the sorted key-value pairs instead that the whole particle array is moved and copied. Furthermore, numerical tests suggest that it is sufficient to reorder the particle array every 100-200 time steps, which can further exploit the temporal coherence mentioned above.

The Z-curve particle reordering algorithm is summarized in a pseudo program as shown in Table 2.

Although particle reordering has to run regularly to adapt the dynamic evolution of SPH simulation, its computational cost almost can be negligible as the Z-values can be computed efficiently from multi-dimensional coordinates using bit interleaving, the almost sorted value-key pairs can be sorted fast, and particularly, only a very small part of particles need to be adjusted each time in the memory position.

Table 2 Pseudo program for Z-curve particle reordering

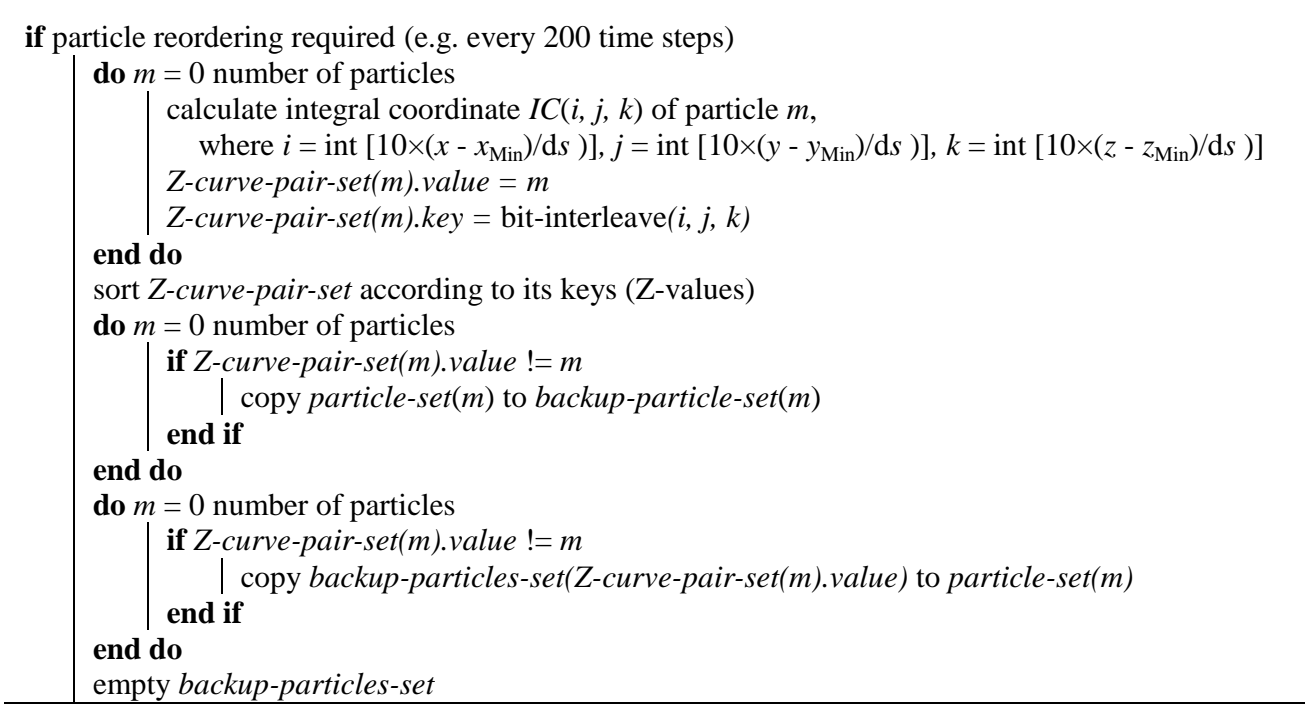




\section{end if}

By preserving spatial locality well, the Z-curve order increase the cache-hit rate and thereby improve the computational efficiency. Figure 4 presents the particle position in memory for a simple two dimensional dam break example. Compared to the circumstance of no particle reordering, data locality is obviously enforced by using Z-curve reordering. The total speedup of the current memory-shared parallelization algorithms is shown in Figure 5 in which the result of the case without particle reordering is also presented for comparison. The scaling of the case without reordering is much worse, while parallelization with particle reordering significantly accelerates the computation with speedup ratio up to 9.7 when 16 threads are used, which is satisfying for memory shared parallelization. The results indicate that particle reordering significantly reduces the memory transfer and thereby improves the performance.
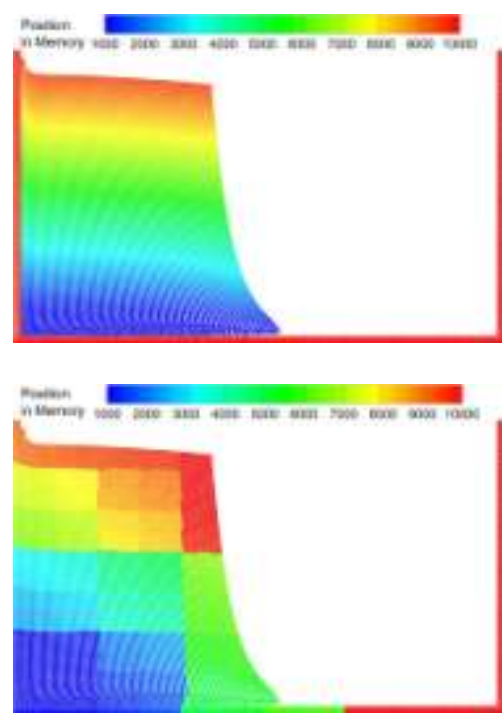

Figure 4 Comparison of particle positions in memory with/without reordering (Particles are colored according their location in memory, where dark blue is the first and red is the last position): (a) Spatial locality is not maintained without particle reordering; (b) Spatial compactness is enforced by Z-curve reordering.

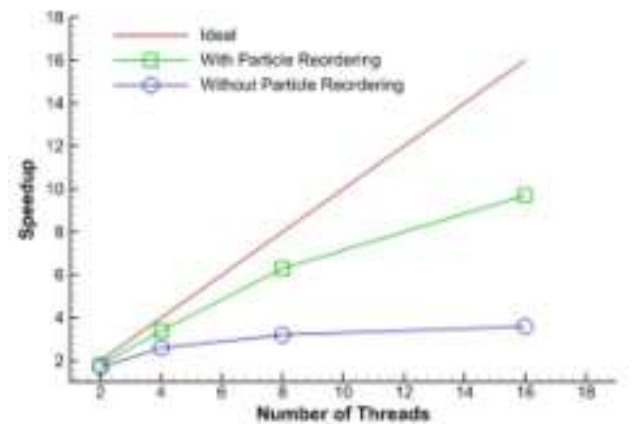


Figure 5 Total speedup of the current memory-shared parallelization algorithm.

\section{Validation and Applications}

\subsection{Two-Dimensional Wedge Water Entry}

The experimental water entry investigation of a wedge [42] is used here as the first case to validate the feasibility and accuracy of the developed SPH method. The wedge geometries and experimental setup can be found in Ref. [42]. The numerical initial conditions for the SPH simulation were defined using the exact velocity and position of the experimental test section at the impact instant. The numerical water tank is $3.6 \mathrm{~m} \times 1.8 \mathrm{~m}$ as shown in Figure 6(a), which is large enough to avoid the unwanted interaction to the wedge caused by the wall boundary reflected sound wave during the concerned time period. The tank was filled with 1620,000 fluid particles with an initial size of $0.002 \mathrm{~m}$. The motion with the velocity profile presented in Figure $6(\mathrm{~b})$, which was recorded in the experiment, was imposed as an input of the simulation. The SPH computation began from the moment when the wedge enters the free surface with a $6.15 \mathrm{~m} / \mathrm{s}$ initial vertical velocity.

(a)

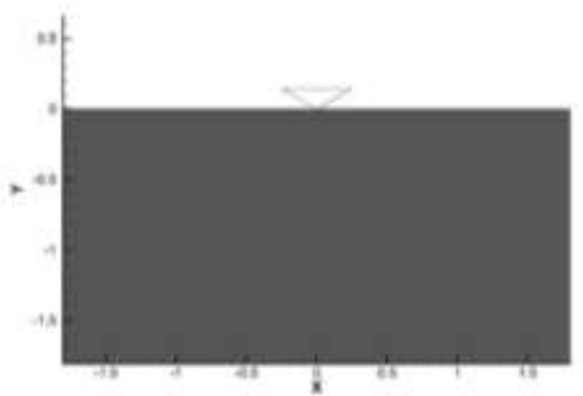

Figure 6 Numerical setup for the water entry case of a 2D wedge: (a) computational domain; (b) verticle velocity profile of the wedge motion.

The influence of the compressibility parameter $B$ on the accuracy is firstly investigated. Three cases with $B=$ $3.60 \mathrm{e}+5 \mathrm{~Pa}, 1.43 \mathrm{e}+6 \mathrm{~Pa}, 5.72 \mathrm{e}+6 \mathrm{~Pa}$, corresponding to reference sound speed of $50 \mathrm{~m} / \mathrm{s}, 100 \mathrm{~m} / \mathrm{s}$ and $200 \mathrm{~m} / \mathrm{s}$, respectively, were simulated where the artificial viscosity model with $\alpha=0.1$ and the predictor-corrector time integration method were employed as the same. The time step size was fixed to $3.5 \mathrm{e}-6 \mathrm{~s}$ for all the three simulations for the sake of consistency. The temporal vertical force exerting on the wedge are shown in Figure 7 where the original force data obtained by the SPH simulations are denoted by thin dash lines while the filtered ones are denoted by thick lines. In the present study, a $1^{\text {st }}$ polynomial order Savitzky-Golay method with 500 points of 
window is employed to obtain smooth curves by filtering original data. The analytical and measured data given by Zhao [42] are also presented in Figure 7 for comparison. A slight overestimation for the SPH simulations is found at the initial stage of the entry. In general, the numerical results by the simulations with reference sound speeds of $100 \mathrm{~m} / \mathrm{s}$ and $200 \mathrm{~m} / \mathrm{s}$ show a good agreement with the experimental data while large deviation can be found for the computation with reference sound speed of $50 \mathrm{~m} / \mathrm{s}$ when $t>0.006 \mathrm{~s}$. This indicates that the reference sound speed of $50 \mathrm{~m} / \mathrm{s}$ is not large enough to keep the density variation in a limited level and thus induces large error. However, too large reference sound speed, e.g. $200 \mathrm{~m} / \mathrm{s}$ in the present case, would induce noisy force generation as shown in Figure 7 due to the fact that the fluid becomes more "rigid" with the increased compressibility parameter $B$ and thus causes stronger numerical noise in density field and oscillations in pressure field. Comparison of the filtered vertical force histories shows only very small variation between the cases with reference sound speed of $100 \mathrm{~m} / \mathrm{s}$ and $200 \mathrm{~m} / \mathrm{s}$, indicating that the averaged force generation is not affected significantly by the increased parameter $B$ when it meets the requirement of not inducing too large density variation.

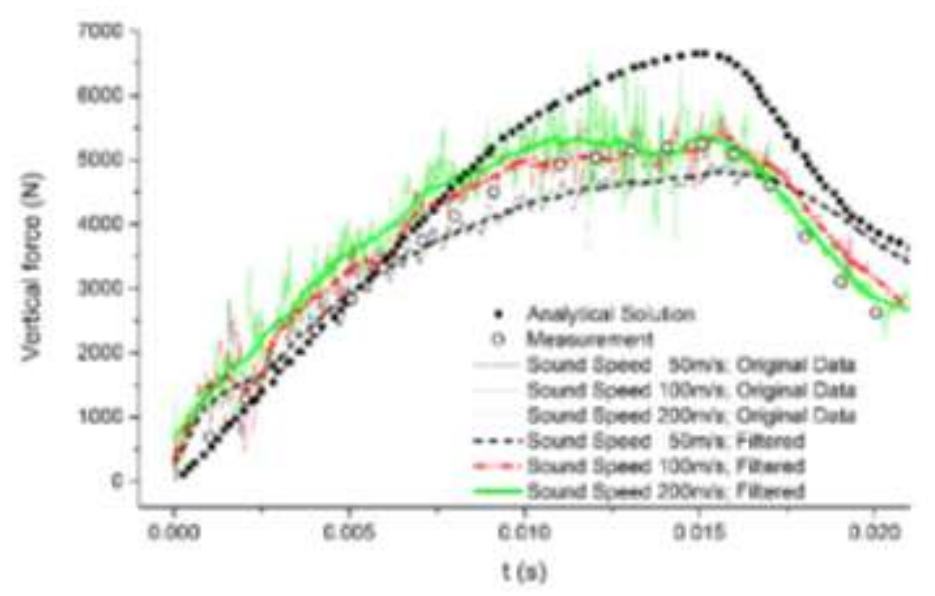

Figure 7 Time histories of vertical forces due to water on the wedge.

Figure 8 shows the velocity and pressure fields at the time instant of $t=0.021 \mathrm{~s}$ computed by using the three different values of parameter $B$. One notable feather related to the wedge water entry is the large deformation of free surface and two splashing flow on the wedge boundaries. No significant difference can be found in the velocity contour and the shape of the two symmetric jets between the three cases as demonstrated in Figure 8(a). The maximum velocity of the jets can reach to more than $20 \mathrm{~m} / \mathrm{s}$, but the velocity of the main fluid near the wedge boundaries is about $6 \mathrm{~m} / \mathrm{s}-10 \mathrm{~m} / \mathrm{s}$, which suggests that reference sound speed of at least $60-100 \mathrm{~m} / \mathrm{s}$ should be used in order to keep error in a low level. It also explains why the vertical force obtained by using reference sound speed of $50 \mathrm{~m} / \mathrm{s}$ deviates more from the measurement than that computed by using $100 \mathrm{~m} / \mathrm{s}$. The pressure contours shown in 
Figure 8(b) indicate obvious disparity in the transmission of pressure wave of which the transmitting speed is determined by the compressibility parameter $B$. At the time instant of $t=0.021 \mathrm{~s}$, the front of the pressure waves reaches to the center region of the computational domain in the case with reference sound speed of $50 \mathrm{~m} / \mathrm{s}$, while it has reached to and has reflected from the wall boundaries in the cases with reference sound speed of $100 \mathrm{~m} / \mathrm{s}$ and $200 \mathrm{~m} / \mathrm{s}$, respectively.

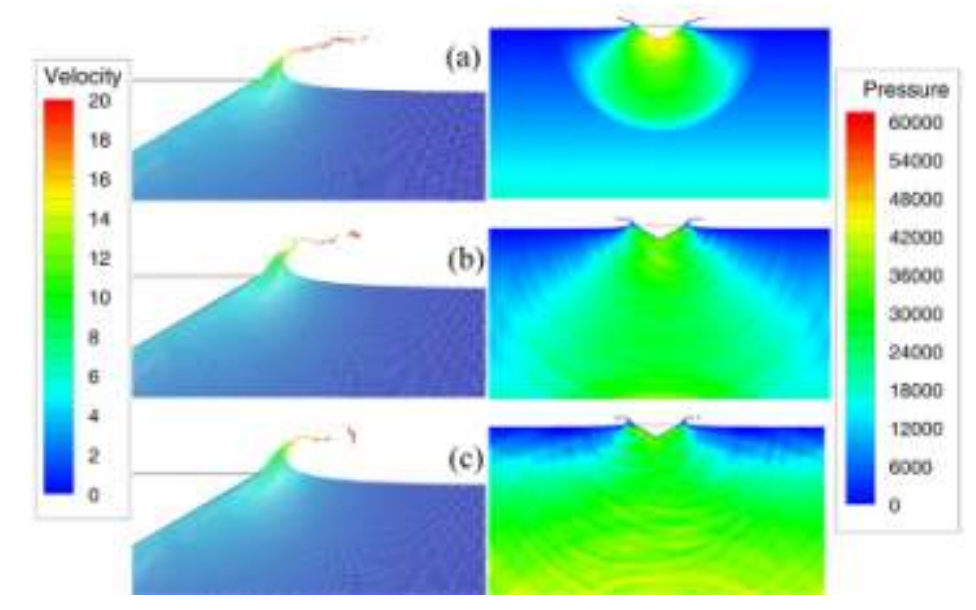

Figure 8 Velocity and pressure field of $2 \mathrm{D}$ wedge entry case at $\mathrm{t}=\mathbf{0 . 0 2 1} \mathrm{s}$ computed with reference sound speed of (a) $50 \mathrm{~m} / \mathrm{s}$, (b) $100 \mathrm{~m} / \mathrm{s}$ and (c) $200 \mathrm{~m} / \mathrm{s}$ (left column: velocity contour; right column: pressure contour).

The influence of viscous models on the simulation is also studied. Artificial viscosity with $\alpha=0.05,0.1,0.2,0.4$ and laminar model were considered. The simulations were performed by using reference sound speed of $100 \mathrm{~m} / \mathrm{s}$, predictor-corrector time integration method and a fixed time step size of 3.5e-6s. The vertical force histories and the deformed free surface and the splashing jet at the time instant of $t=0.021 \mathrm{~s}$ are compared in Figure 9 where the results of the case with artificial viscosity of $\alpha=0.05$ are not presented as the computation of this case was not performed successfully due to numerical instability caused by the insufficient artificial viscosity. When comparing the vertical force in general as shown in Figure 9(a), all the results obtained by artificial viscosity or laminar model agree well with experimental measurement. The curve of the vertical force computed by artificial viscosity with larger $\alpha$ (e.g. 0.4) is more smooth than the other smaller ones, indicating that increasing artificial viscosity is beneficial to numerical stability. However, the increased artificial viscosity makes the fluid jet shorter (see Figure 9(b)) as the fluid could be dragged more by the increased viscous force. Laminar model slightly overestimates the vertical fore comparing with those of artificial viscosity and with experimental data when $t<0.016 \mathrm{~s}$. 
Figure 10 represents the vertical force histories and zoomed-in splashing jet at time instant of $t=0.021 \mathrm{~s}$ computed by using predictor-corrector and Runge-Kutta time integration methods. The three simulations were carried out by using reference sound speed of $100 \mathrm{~m} / \mathrm{s}$, artificial viscosity model with $\alpha=0.2$ and variable time step size calculated according to CFL condition. As noted in Figure 10(a), the three integration methods provide similar results in force generation. The force curve obtained by 2-stage Runge-Kutta integration, however, shows less smooth than the other two, and 4-stage Runge-Kutta integration slightly superiors to predictor-corrector method in accuracy comparing to the measurement but at the expense of almost two times computational cost. No significant difference can be found in the shape and position of the splashing jet computed by the three time integration methods as demonstrated in Figure 10(b).

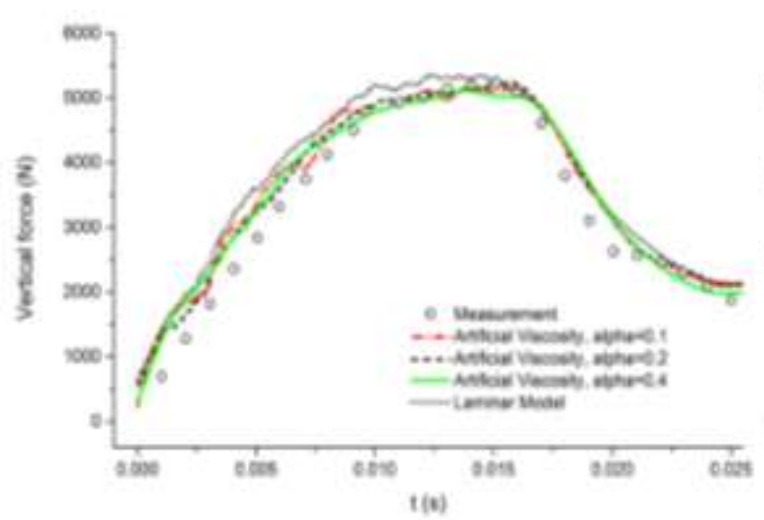

(a)

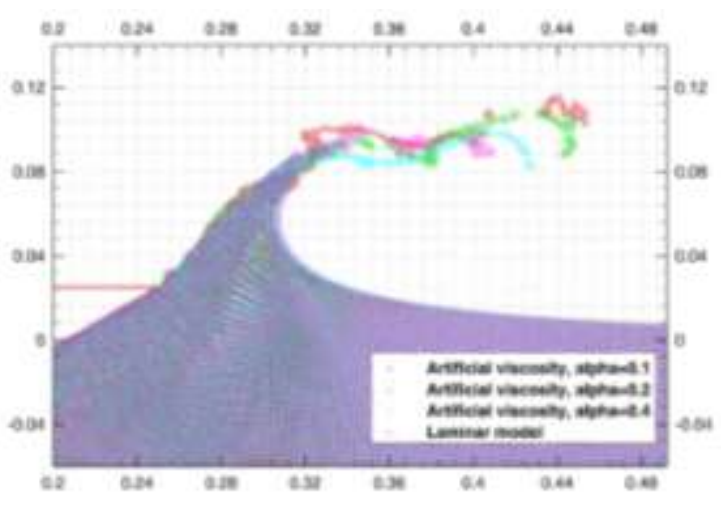

(b)

Figure 9 Vertical force histories and splashing jet obtained by artificial viscosity and laminar model: (a) vertical force histories; (b) zoomed-in splashing jet.

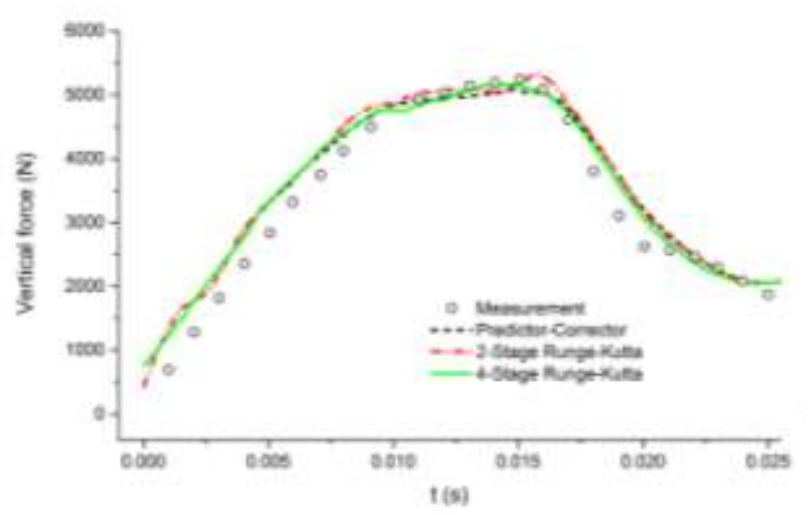

(a)

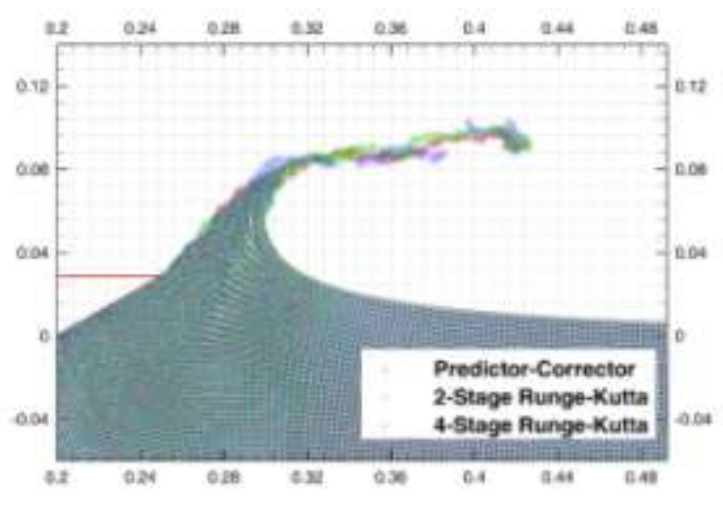

(b)

Figure 10 Vertical force histories and splashing jet obtained by using predictor-corrector and Runge-Kutta time integration methods: (a) vertical force histories; (b) zoomed-in splashing jet. 


\subsection{Three-dimensional Dam-break}

This section presents a three-dimensional free-surface dam-break flow impacting on a tall vertical square column structure with much smaller width comparing to the tank, indicating a flow evolution with strong threedimensional effects. The resulting velocities and the forces exerted by the waves on the structure will be compared with the experimental data from Yeh and Petroff's experiment (The data can be accessible from the study of Marrone and Antuono [43]). The schematics of the experimental setup is shown in Figure 11, they utilized a rectangular tank of $1.6 \mathrm{~m} \times 0.61 \mathrm{~m} \times 0.75 \mathrm{~m}$. A volume of water (with $0.61 \mathrm{~m} \times 0.4 \mathrm{~m} \times 0.3 \mathrm{~m}$ ) is initially contained behind the gate before being released. Besides, a thin layer of water (1.0 $\mathrm{cm}$ deep) was also presented in the tank downstream of the gate. The experiment measured the time histories of the force on the structure and the velocity at the location of $14.6 \mathrm{~cm}$ upstream of the structure center and $2.6 \mathrm{~cm}$ off the tank floor.

The numerical simulation was performed with a 4-stage Runge-Kutta algorithm and an artificial viscosity treatment with $\alpha=0.01$. Constant parameter $B$ was set at $3.60 \mathrm{e}+5 \mathrm{~Pa}$, corresponding to a reference sound speed of $50 \mathrm{~m} / \mathrm{s}$ that is large enough to ensure the density variation less than $1 \%$. Fluid particles were initially placed with zero initial velocity and with a gravity pressure. The initial particle spacing was chosen to be $0.005 \mathrm{~m}$ resulting in total 1.33 million particles. Total $1.6 \mathrm{~s}$ of physical time was simulated with variable time step size at the order of $1.0 \mathrm{e}-5 \mathrm{~s}$.

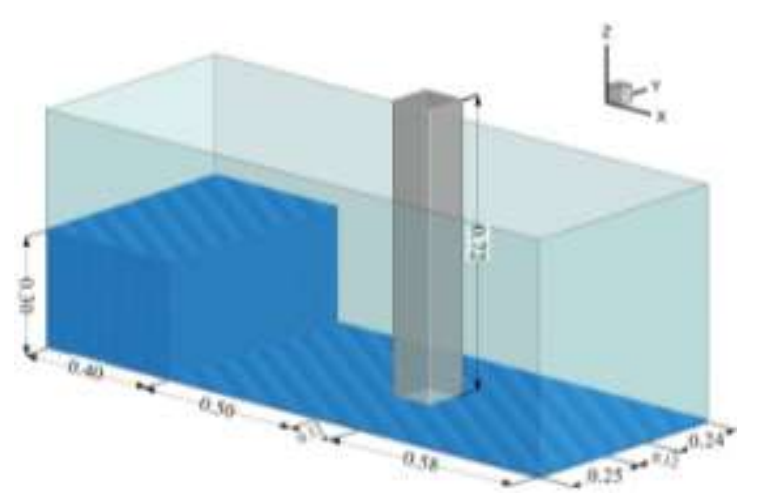

Figure 11 Schematic diagram of the experimental setup

Figure 12 represents the time histories of the force along $x$-axis acting on the structure and of the velocity at the measurement location mentioned above. Comparing with the experimental data, both the velocity and the force are observed to have a good agreement. The amplitude of the first peak, the variation trends with time are well predicted as well as the inflection point of the force direction, indicating that the developed SPH code can provide 
satisfactory results for predicting the dynamic response of complex free surface flows.

Figure 13 show the snapshots of wave propagation in collision with the structure at the time of $0.5514 \mathrm{~s}$ and $1.3344 \mathrm{~s}$, in perspective, top and side views, respectively, which clearly illustrate the strong three-dimensional effect of the flow. As can be seen from Figure 13 ( $t=0.5514 \mathrm{~s}$ ), the impacting of wave on the column divides the upstream flow into two streams and results in a horse shoe pattern flow around the structure. Then, moving to $t=1.3374 \mathrm{~s}$, the upstream flow separating around the structure interacts with the downstream flow which comes back after hitting the right vertical wall. Due to obstructing of the column to the upstream flow, the interaction at the two sides of the tank is stronger than that in the middle, leading to an irregular shape of back flow.

(a)

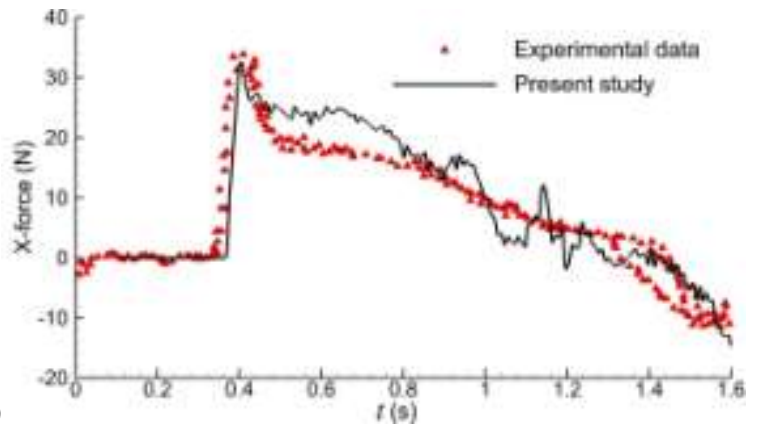

(b)

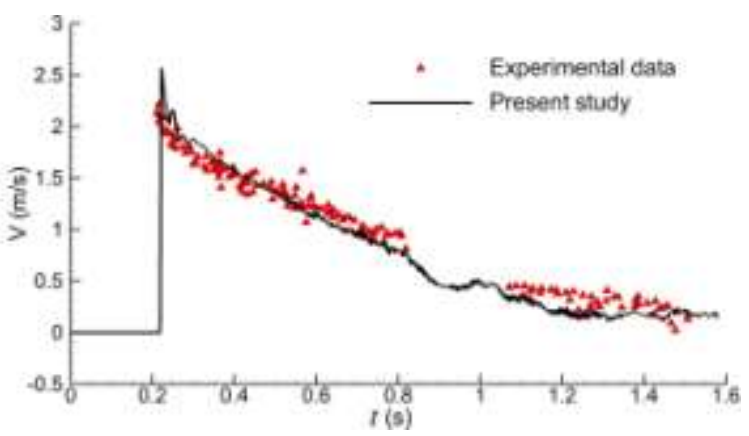

Figure 12 Comparison of SPH computational results to the experimental data: (a) x-force; (b) velocity.
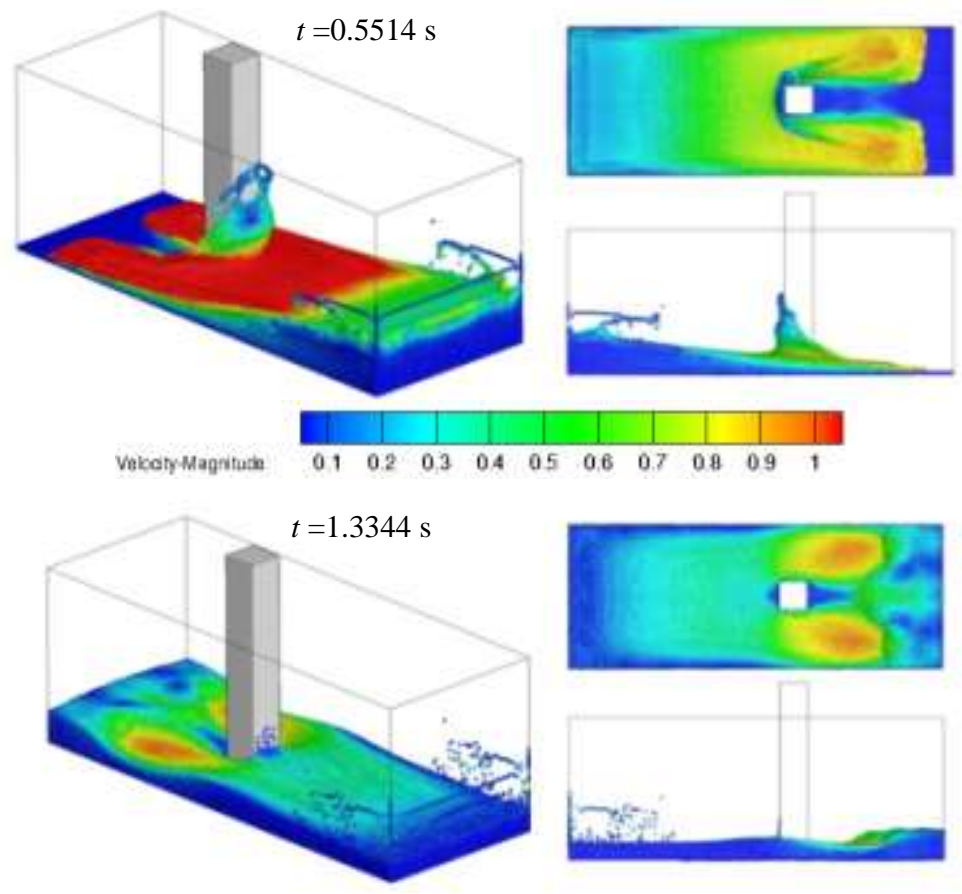

Figure 13 Snapshots of wave propagation in collision with the column at the time of $0.5514 \mathrm{~s}$ and $1.3344 \mathrm{~s}$. 


\subsection{Ditching of the Fuselage of NACA TN2929 Model}

In this section, the ditching characteristics of the NACA TN 2929 model [44] are simulated using the developed SPH code. McBride and Fisher [44] experimentally investigated the effect of rear-fuselage shape of NACA TN2929 model on ditching characteristics in NASA Langley research center. Nine different models were studied in their measurement campaign, and temporal histories of forward velocity, pitching attitude and gravity center height above the water were recorded. The simulation was performed on the model A (see Figure 14(a)) from MacBride and Fisher [44] with an initial incident angle and landing speed at $10 \mathrm{deg}$ and $9.14 \mathrm{~m} / \mathrm{s}$ respectively. In the present study, the aerodynamic effect is ignored as the aerodynamic load on this pure fuselage is not thought to have significant effect on its dynamics with the lack of wings. Results are compared with the experimental data to evaluate the capability of simulating aircraft ditching of the developed SPH code. The surface mesh of the model and the dummy particles can be seen in Figure 14(b) and (c), while Table 3 shows the details of the computational setup for the simulation. The domain of the water at rest was set at $4 \mathrm{~m} \times 2 \mathrm{~m} \times 0.875 \mathrm{~m}$. Moreover, the distance between the particles is $0.0125 \mathrm{~m}$, and the time step size is around $2.25 \times 10^{-5} \mathrm{~s}$. The simulation was performed by using the laminar viscosity treatment and the predictor-corrector time integration. Note that a $0.2 \mathrm{~m} / \mathrm{s}$ initial vertical velocity was introduced to guarantee the consistency for the simulation and the experiment since the model was released at a certain height above the water surface in the experiment while the simulation started from the position right at the water level.

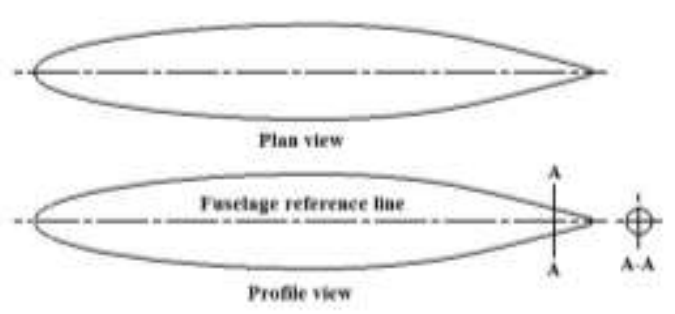

(a)

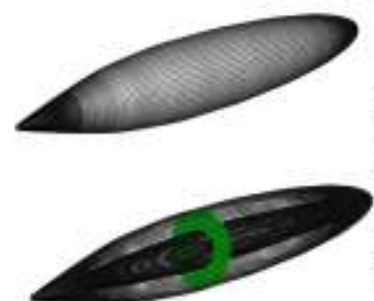

(b)

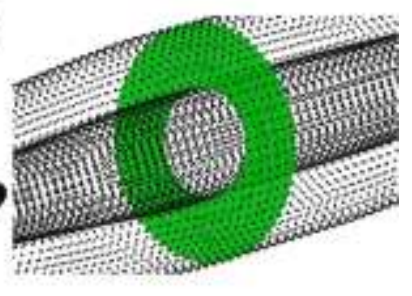

(c)

Figure 14 NACA TN-2929 model and the mesh system: (a) geometry of the tested model (adapted from McBride and Fisher [44]; (b) surface mesh of the tested model; (c) zoom view and the dummy particles.)

Table 3 Computational setup for the NACA TN-2929 model

\begin{tabular}{cc}
\hline \hline Computational Setup & Value \\
\hline Length and width & $1.22 \mathrm{~m}, 0.104 \mathrm{~m}$ \\
Mass & $5.6699 \mathrm{~kg}$ \\
$I_{x x} I_{y y} I_{z z}$ & $0.2924 \mathrm{~kg} \cdot \mathrm{m}^{2}, 0.2924 \mathrm{~kg} \cdot \mathrm{m}^{2}, 0.5263 \mathrm{~kg} \cdot \mathrm{m}^{2}$
\end{tabular}




$\begin{array}{cc}\text { Forward velocity } & 9.144 \mathrm{~m} / \mathrm{s} \\ \text { Vertical velocity } & 0.2 \mathrm{~m} / \mathrm{s} \\ \text { Incident angle } & 10 \mathrm{deg}\end{array}$

Figure 15 presents the ditching behavior of the NACA TN-2929 model at different time instants in terms of pitching angle and forward velocity. An obvious pitching-down and pitching-up motion (seen in Figure 16) can be observed in Figure 15(a) where the simulated pitching behavior shows a good agreement with the experimental data in general. The model demonstrates a nose-down pitching during the initial impact with the simulation slightly overestimating the pitching-down motion before the time of $0.1 \mathrm{~s}$. The notable pitching-up motion after $0.1 \mathrm{~s}$ with the pitching angle up to 35 degree, which was predicted accurately comparing with the experiment data, indicates a significant pitching up moment caused by rear suction forces. The forward velocity history shown in Figure 15(b) illustrates a gradually decelerating after the water impact which is a realistic behavior. However, comparing against the test data, the simulation result shows a less strong deceleration in forward velocity. Such an underestimation can be found similarly in the work of Streckwall et al [45] and Qu et al [16] where they ascribed the difference to the low precision of the experiment carried out in 1956.

(a)

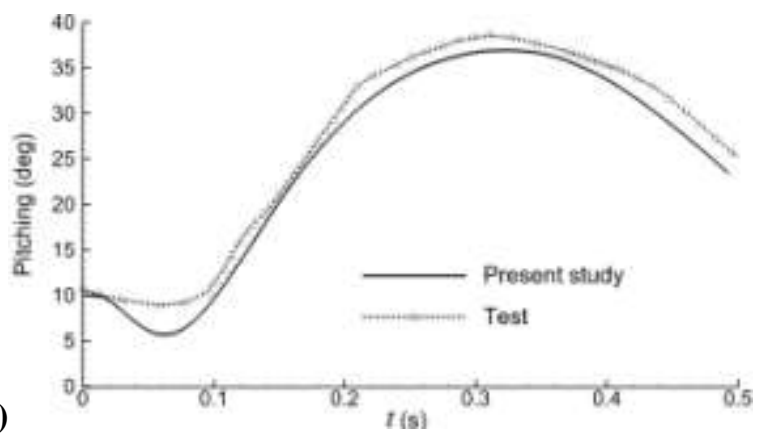

(b)

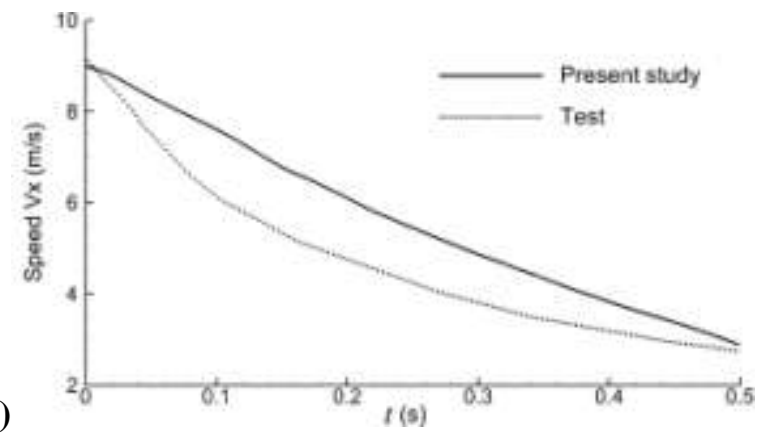

Figure 15 Ditching motion histories and the comparison with the experimental data [44]: (a) pitching angle;

\section{(b) forward speed.}

Figure 16 shows the model attitude and water surface deformation during ditching at different time instants, viz. $0.05 \mathrm{~s}, 0.1 \mathrm{~s}, 0.2 \mathrm{~s}, 0.3 \mathrm{~s}, 0.4 \mathrm{~s}$ and $0.5 \mathrm{~s}$, and the wetted surface pressure contours of the model at the corresponding time instants are presented in Figure 17. The pressure contours show a high pressure region at the front part of the wetted surface and a negative pressure region (respect to the reference pressure) at the rear part of the model. Due to the convex-curved shape of the model, the impact of the model accelerate the water and water pressure decreases, resulting in a negative pressure region. When the negative pressure region is dominating, suction force will be 
generated and load on the rear fuselage, leading to a pitching up motion as can be seen from Figure $\mathbf{1 6}$ and the pitching-angle history in Figure 15(a).
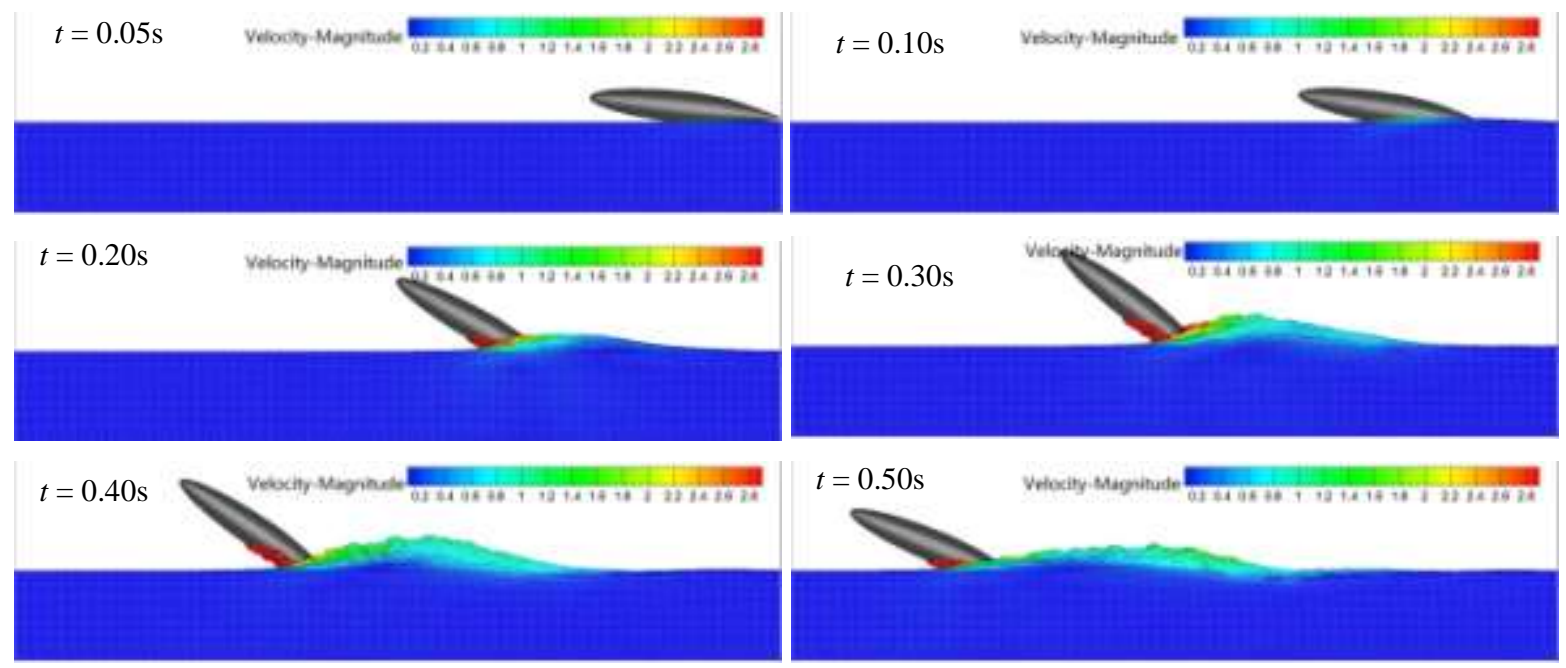

Figure 16 Attitude of NACA TN-2929 model and water surface deformation during ditching at different time instants, viz. $0.05 \mathrm{~s}, 0.1 \mathrm{~s}, 0.2 \mathrm{~s}, 0.3 \mathrm{~s}, 0.4 \mathrm{~s}$, and $0.5 \mathrm{~s}$.

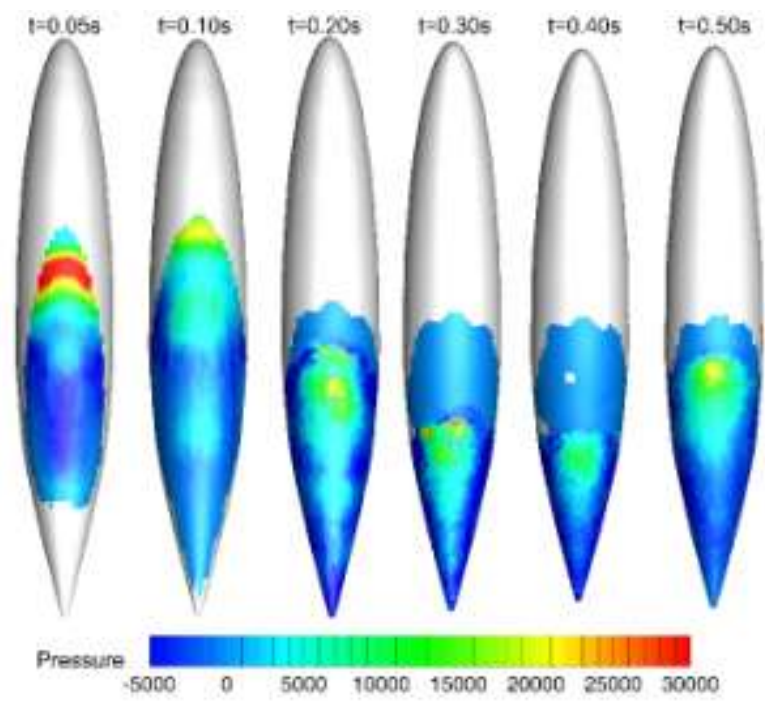

Figure 17 Pressure distribution on the bottom surface of NACA-TN2929 model during ditching at different time instants, viz. $0.05 \mathrm{~s}, 0.1 \mathrm{~s}, 0.2 \mathrm{~s}, 0.3 \mathrm{~s}, 0.4 \mathrm{~s}$, and $0.5 \mathrm{~s}$.

\subsection{Helicopter Ditching Application}

This section applies the developed SPH method to a complex scaled-down (1/8) helicopter (seen in Figure 18) ditching on the water. Note that, the rotors are removed for the sake of simplification. Detailed information of the geometry and computational setup can be found in Figure 18 and Table 4. The water domain is $4 \mathrm{~m} \times 2 \mathrm{~m} \times 0.875 \mathrm{~m}$. The distance of the particles is set at $0.0125 \mathrm{~m}$, resulting in a total of 3.384 million particles. The artificial viscosity 
was treated with $\alpha=0.01$. 4-stage Runge-Kutta integration with variable time step size was used for this application. The constant $B$ is chosen as $6 \times 10^{6} \mathrm{~Pa}$ which is large enough to limit the density variation. To evaluate the effect of ditching angle on the helicopter, three ditching scenarios with different incident angle, viz. 6deg, 8deg and 10 deg, were simulated.

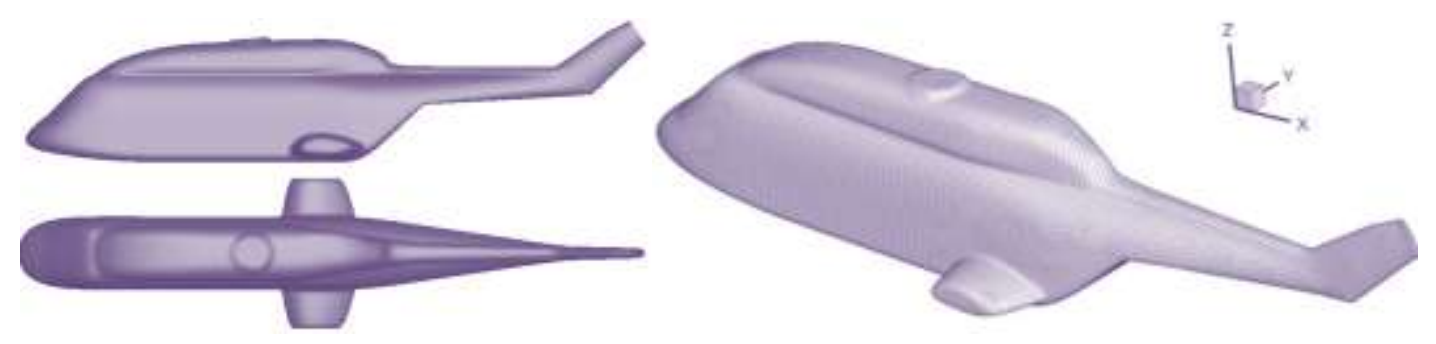

Figure 18 CAD model and the computational mesh of the helicopter.

Table 4 Computational setup for the helicopter model

\begin{tabular}{cc}
\hline \hline Parameters & Value \\
\hline Fuselage Length & $2.4 \mathrm{~m}$ \\
Fuselage Width & $0.574 \mathrm{~m}$ \\
Mass & $25.9 \mathrm{~kg}$ \\
$I_{x x}, I_{y y} I_{z z}$ & $1.21 \mathrm{~kg} \cdot \mathrm{m}^{2}, 4.58 \mathrm{~kg} \cdot \mathrm{m}^{2}, 0.46 \mathrm{~kg} \cdot \mathrm{m}^{2}$ \\
$I_{x y}, I_{y z}, I_{x z}$ & $4.12 \mathrm{~kg} \cdot \mathrm{m}^{2}, 0.01 \mathrm{~kg} \cdot \mathrm{m}^{2}, 0.01 \mathrm{~kg} \cdot \mathrm{m}^{2}$ \\
Vertical speed & $0.5033 \mathrm{~m} / \mathrm{s}$ \\
Incident angle & $6,8,10 \mathrm{deg}$ \\
\hline \hline
\end{tabular}

Figure 19 shows the vertical and horizontal accelerations of the helicopter model with respect to the time for different incident angles. As can be seen, the helicopter experiences positive accelerations during ditching. A notable increase in both vertical and horizontal acceleration is revealed from $0.04 \mathrm{~s}$ to $0.06 \mathrm{~s}$, when the ditching just happens. The peaks of the vertical acceleration for the simulated three different incident angles are $2.46 \mathrm{~g}, 2.03 \mathrm{~g}$ and 1.61g, respectively, showing decrease with the increased incident angle; while the peak of the horizontal acceleration increases when the initial incident angle is increased.

The pitching angle and angular velocity history for three different incident angles are plotted in Figure 20(a) and (b), respectively. As expected, a pitching down and pitching up motion is found in all the three cases with different ditching incident angles. The rear-fuselage part is first impacted with the water, where a pitching down moment is created with respect to the center of gravity. After a short time, the water moves with the helicopter due to the interaction between the water and the immersed fuselage. As some pressure energy of the water is converted into kinetic energy, a drop of water pressure is expected, hence resulting in a region of negative pressures with 
respect to the ambient pressure at the rear-fuselage which can produce a pitching up moment. The pitching up motion of the helicopter can be revealed after $t=0.15 \mathrm{~s}$ as referring to Figure 20 (a).

(a)
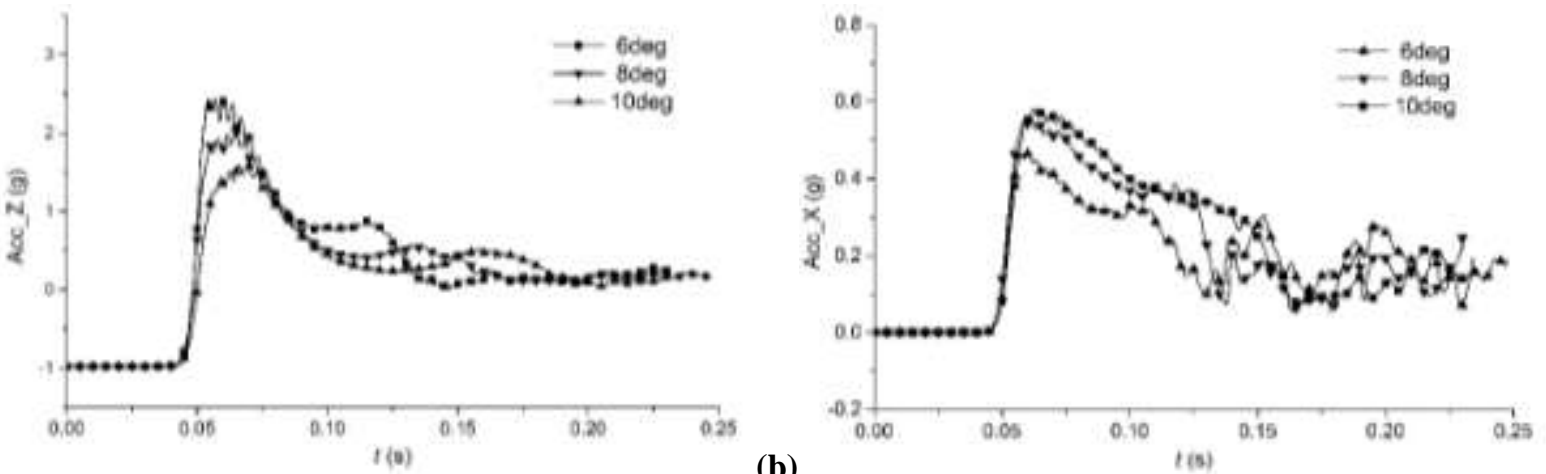

(b)

Figure 19 Acceleration history of the helicopter in water ditching: (a) vertical acceleration; (b) horizontal acceleration.

(a)

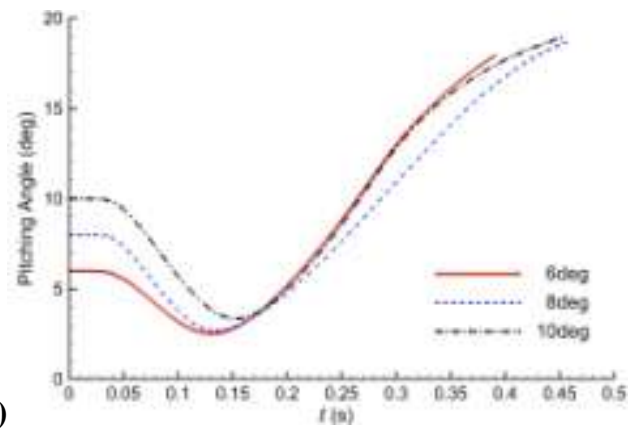

(b)

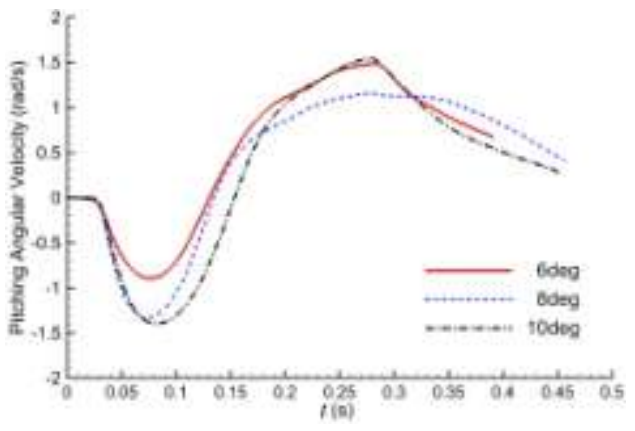

Figure 20 Pitching angle (a) and angular velocity history (b) during the helicopter model ditching.

In order to give a better understanding of the ditching event, the pressure contours at the wetted belly of the helicopter at two time instants (i.e., $t=0.04 \mathrm{~s}$ and $0.081 \mathrm{~s}$ ) are plotted in Figure 21. At $t=0.04 \mathrm{~s}$ when the helicopter experiences the largest acceleration as shown in Figure 19(a), an overpressure region dominates the wetted area of the helicopter belly and only a very small area of negative pressure exists at the kinking part of the belly. The incident angle at $8 \mathrm{deg}$ has the highest pressure contour with a peak at about $60000 \mathrm{~Pa}$, which conforms to the acceleration behavior shown in Figure 19. Moving to the time of $t=0.081 \mathrm{~s}$, the high pressure region moves forward while a notable low pressure region is found at the rear-fuselage. Such a low pressure region creates rear suction and induces a pitching up motion of the helicopter as shown in Figure $\mathbf{2 0}$ when the suction force is strong enough.

The nose-down and nose-up pitching motion caused by hydrodynamic effects of overpressure and suction can be further demonstrated in Figure 22 where the attitude of the model at different time instants during ditching with 6deg incident angle are presented as well as the deformed free surface of water near the model. The particles on 
water free surface are colored by velocity magnitude. A nose-down pitching can be firstly observed at the initial stage of the impacting on water then followed by a gradual nose-up pitching after $0.21 \mathrm{~s}$. The impact of the front fuselage and the stub wing leading edge on the water causes a significant splashing jet, while the fluid flow around the convex-curved rear fuselage can be also revealed which is regarded as the result of the sticky contact of the wall surface and the water modeled by the dummy and fluid particles.

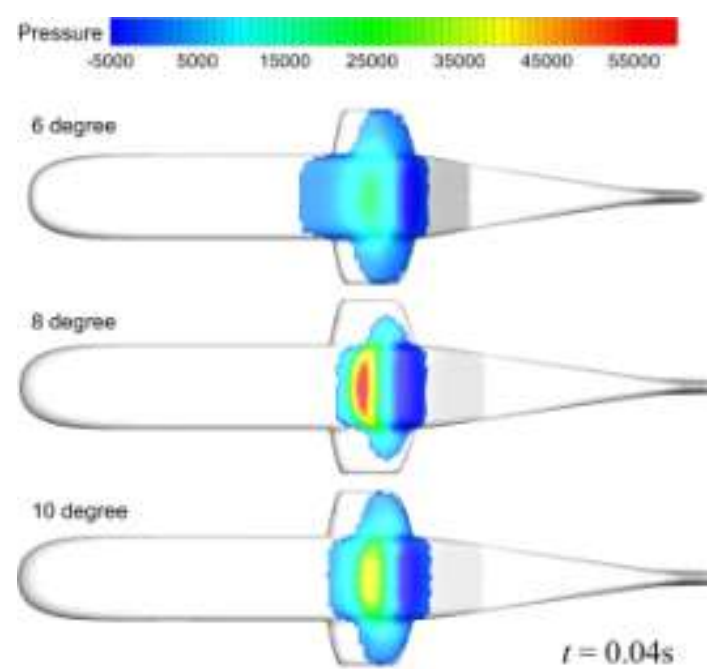

(a)
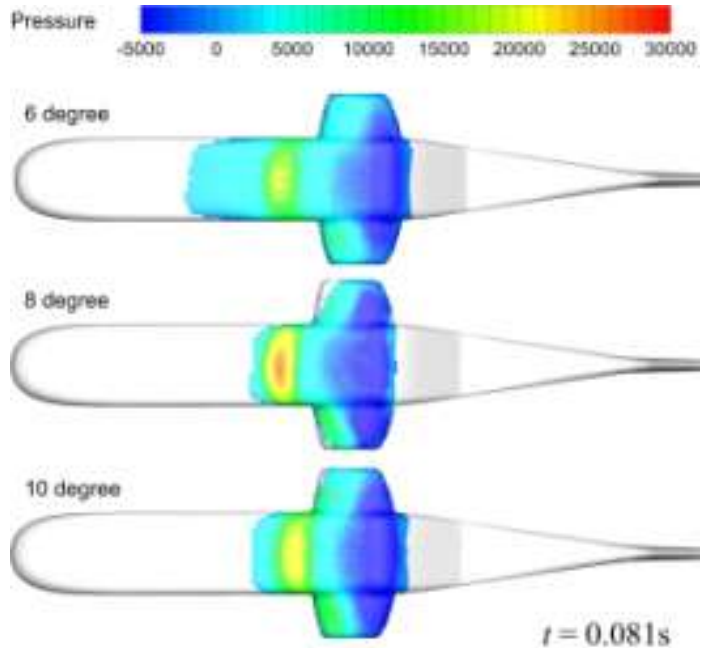

(b)

Figure 21 Pressure contour of the wetted belly of the helicopter model: (a) $t=0.04 \mathrm{~s}$; (b) $t=0.081 \mathrm{~s}$.

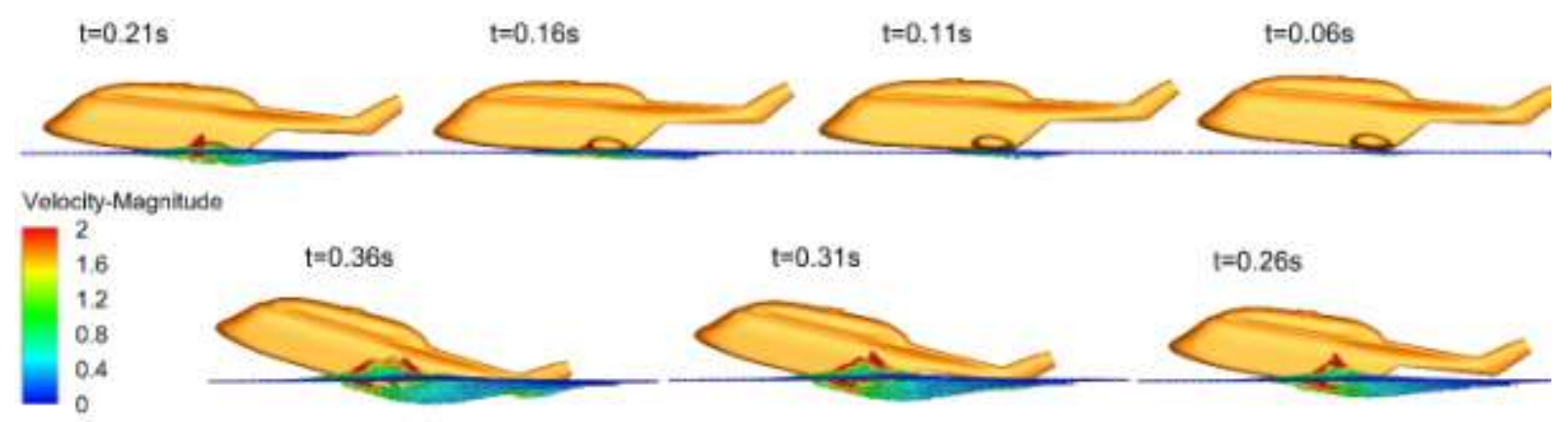

Figure 22 Attitude of the helicopter model during ditching with incident angle of 6 degree.

\section{Conclusion}

A weakly compressible SPH method coupled with six-degree-of-freedom dynamics was developed in the present study for the simulation of aircraft ditching events. A neighbor particle search scheme and memory-shared parallelization with Z-curve particle reordering were highlighted which can accelerate the computation significantly. 
For the sake of accurate prediction of hydrodynamic effects such as overpressure and suction, a simple dummy particle wall boundary treatment method was also developed with the dummy particles automatically generated for three-dimensional complex geometries. Several typical applications including a two-dimensional wedge entry, a three-dimensional dam break and a NACA TN-2929 model ditching were employed as validation cases in which the effects of the compressibility parameter, viscous models and time integration methods on accuracy or efficiency were also investigated. The computational results in the present study indicate a good agreement in general with the documented experimental results. The developed SPH method was successfully applied to predict the ditching event of a complex helicopter model with varying ditching angles. The computational results reveal the associated hydrodynamics and dynamics of the helicopter during ditching.

\section{Acknowledgments}

This work has been supported by the National Science Foundation of China (No. 11672133) and the Project of Helicopter Ditching Analysis and Test (No. 2011DFR80210). The support from China Scholarship Council (CSC, No. 201303070173) is also appreciated.

\section{References}

[1] Pentecote N. "Validation of PAM-CRASH Code for the Simulation of the Impact on Water," DLR-IB 435.2003/3, Jan. 2003.

[2] Pentecote, N., and Vigliotti, A., "Crashworthiness of Helicopters on Water: Test and Simulation of a Full-scale WG30 Impacting on Water,” International Journal of Crashworthiness, Vol. 8, 2003, pp. 559-572.

[3] Climent, H., Benitez, L., Rosich F., Rueda, F. and Pentecote N. "Aircraft Ditching Numerical Simulation", 25th International Congress of the Aeronautical Sciences (ICAS), Hamburg, Germany, 2006.

[4] Iafrati, A., Grizzi, S., Siemann, M.H., and Benítez Montañés, L., "High-Speed Ditching of a Flat Plate: Experimental Data and Uncertainty Assessment,” Journal of Fluids and Structures, Vol.55, 2015, pp. 501-525.

[5] von Karman, T., “The Impact on Seaplane Floats During Landing,” National Advisory Committee for Aeronautics, 1929.

[6] Wagner, H., "Phenomena Associated with Impact and Gliding on a Liquid Surface," NACA Translation, 1932.

[7] Farhat, G., “Analytical Method for the Ditching Analysis of an Airborne Vehicle,” Journal of Aircraft, Vol. 27, 1989, pp. 312-319.

[8] Reinhard, M. V., "Free Elastic Plate Impact into Water,” PhD thesis, University of East Anglia, 2013. 
[9] Vignjevic, R., and Meo, M., "Simulation of Helicopter Under-floor Structure Impact on Water," International Journal of Crashworthiness, Vol. 6, 2001, pp. 425-443.

[10] Ortiz, R., Portemont, G., Charles, J.L., and Sobry, J.F., “Assessment of Explicit FE Capabilities for Full Scale Coupled Fluid/Structure Aircraft Ditching Simulations," International Council of the Aeronautical Sciences, Toronto, Canada, 8-13 September, 2002.

[11] Jackson, K.E., and Fuchs, Y.T., "Comparison of ALE and SPH Simulations of Vertical Drop Tests of a Composite Fuselage Section into Water," 10th International LS-DYNA Users Conference, Dearborn, United States, 8-10 June, 2008.

[12] Hua, C., Fang, C., and Cheng, J., "Simulation of Fluid-Solid Interaction on Water Ditching of an Airplane by ALE Method," Journal of Hydrodynamics, Vol. 23, 2011, pp. 637-642.

[13] Hu, W., Wang, Y.H., and Chen, C.H., "Numerical Simulation of Aircraft Ditching Based on ALE Method," Applied Mechanics and Materials, Vol. 668-669, Nov. 2014, pp. 490-493.

[14] Wick, A., "Computational Simulation of an Unmanned Air Vehicle Impacting Water," PhD thesis, Iowa State University, 2006.

[15] Guo, B., Liu, P., Qu, Q., and Wang, J., "Effect of Pitch Angle on Initial Stage of a Transport Airplane Ditching," Chinese Journal of Aeronautics, Vol. 26, 2013, pp. 17-26.

[16] Qu, Q., Hu, M., Guo, H., Liu, P., and Agarwal, R.K., "Study of Ditching Characteristics of Transport Aircraft by Global Moving Mesh Method," Journal of Aircraft, Vol.52, No.5, 2015, pp.1550-1558.

[17] Monaghan, J. J., "Smoothed Particle Hydrodynamics," Annual Review of Astronomy and Astrophysics, Vol. 30, Sep. 1992, pp. 543-574.

[18] Monaghan, J. J., "Simulating Free Surface Flows with SPH,” Journal of Computational Physics, Vol. 110, 1994, pp. 399406.

[19] Shao, S., and Lo, E. Y. M., "Incompressible SPH Method for Simulating Newtonian and Non-Newtonian Flows with a Free Surface,” Advances in Water Resources, Vol. 26, July. 2003, pp. 787-800.

[20] Ellero, M., Serrano, M., and Espanol, P., "Incompressible Smoothed Particle Hydrodynamics," Journal of Computational Physics, Vol. 226, 2007, pp. 1731-1752.

[21] Nair, P., and Tomar, G., “An Improved Free Surface Modeling for Incompressible SPH,” Computers \& Fluids, Vol. 102, 2014, pp. 304-314.

[22] Gomez-Gesteira, M., Rogers, B. D., Crespo, A. J. C., Dalrymple, R. A., Narayanaswamy, M., and Dominguez, J. M., “SPHysics - Development of a Free-Surface Fluid Solver - Part 1: Theory and Formulations," Computers \& Geosciences, Vol. 48, 2012, pp. 289-299. 
[23] Gomez-Gesteira, M., Crespo, a. J. C., Rogers, B. D., Dalrymple, R. a., Dominguez, J. M., and Barreiro, a., "SPHysics Development of a Free-Surface Fluid Solver - Part 2: Efficiency and Test Cases,” Computers \& Geosciences, Vol. 48, 2012, pp. 300-307.

[24] Barreiro, A., Crespo, A. J. C., Dominguez, J. M., and Gomez-Gesteira, M., "Smoothed Particle Hydrodynamics for Coastal Engineering Problems," Computers \& Structures, Vol. 120, 2013, pp. 96-106.

[25] Groenenboom, P. H. L., and Cartwright, B. K., "SPH Simulations of Free Surface Waves and the Interaction with Objects," V European Conference on Computational Fluid Dynamics ECCOMAS CFD, Lisbon, Portugal, 14-17 June, 2010.

[26] Cartwright, B. K., Chhor, A., and Groenenboom, P. H. L., "Numerical Simulation of a Helicopter Ditching with Emergency Flotation Devices," 5th International SPHERIC Workshop, Manchester, UK, 22-25 June, 2010.

[27] Groenenboom, P. H., Campbell, J., and Bentez, L. "Innovative SPH Methods for Aircraft Ditching," Proceed of 11th WCCM/5th ECCM, Barcelona, Spain. 2014.

[28] Groenenboom, P. H. "SPH for Two-Phase Fluid Flow Including Cavitation," Proceed of 7th International SPHERIC Workshop, Prato, Italy, 2012.

[29] Benítez Montanes, L., Climent Manez, H., Siemann, M.H., and Kohlgrueber, D., "Ditching Numerical Simulations: Recent Steps in Industrial Applications," Aerospace Structural Impact Dynamics International Conference, Wichita, USA, 6-9 November, 2012.

[30] Groenenboom, P. H. and Seimann, M. H., "Fluid-Structure Interaction by the Mixed SPH-FE Method with Application to Aircraft Ditching”, International Journal of Multiphysics, Vol.9, No.3, 2015, pp.249-265.

[31] Grimaldi, A., Benson, D.J., Marulo, F., and Guida, M., "Steel Structure Impacting onto Water: Coupled Finite Element Smoothed Particle Hydrodynamics Numerical Modeling," Journal of Aircraft, Vol. 48, No.4, 2011, pp. 1299-1308.

[32] Hughes, K., Vignjevic, R., Campbell, J., De Vuyst, T., Djordjevic, N., and Papagiannis, L., "From Aerospace to Offshore: Bridging the Numerical Simulation Gaps-Simulation Advancements for Fluid Structure Interaction Problems,” International Journal of Impact Engineering, Vol. 61, 2013, pp. 48-63.

[33] Hu, X.Y, and Adams, N.A., “A Multi-phase SPH Method for Macroscopic and Mesoscopic Flows," Journal of Computational Physics, Vol. 213, No. 2, 2006, pp. 844-861.

[34] Monaghan, J. J., “SPH without a Tensile Instability,” Journal of Computational Physics, Vol.159, 2000, pp.290-311.

[35] Gray, J. P., Monaghan, J. J., and Swift, R. P., "SPH Elastic Dynamics", Computer Methods in Applied Mechanics and Engineering, Vol.190, No.49-50, 2001, pp.6641-6662. 
[36] Adami, S., Hu, X.Y., and Adams, N.A., “A Generalized Wall Boundary Condition for Smoothed Particle Hydrodynamics,” Journal of Computational Physics, Vol. 231, 2012, pp. 7057-7075.

[37] Monaghan, J.J., "On the Problem of Penetration in Particle Methods," Journal of Computational Physics, Vol. 82, 1989, pp. $1-15$.

[38] Monaghan, J.J., and Kos, A., "Solitary Waves on a Cretan Beach," Journal of Waterway, Port, Coastal, and Ocean Engineering, Vol. 125, 1999, pp. 145-155.

[39] Viccinone, G., Bovolin, V., and Carratelli, E. P., "Defining and Optimizing Algorithms for Neighbouring Particle Identification in SPH Fluid Simulations,” International Journal for Numerical Methods in Fluids, Vo1. 58, 2008, pp. 625-638.

[40] Domínguez, J.M., Crespo, A. J. C., Gómez-Gesteira, M., and Marongiu, J. C., "Neighbour Lists in Smoothed Particle Hydrodynamics," International Journal for Numerical Methods in Fluids, Vol. 67, 2011, pp. 2026-2042.

[41] Lohner, R., “Some Useful Renumbering Strategies for Unstructured Grids,” International Journal for Numerical Methods in Engineering, Vol. 36, 1993, pp. 3259-3270.

[42] Zhao, R., Faltinsen, O., and Aarsnes, J., "Water Entry of Arbitrary Two-dimensional Sections with and without Flow Separation,” 21th Symposium on Naval Hydrodynamics, 1997.

[43] Marrone, S., Antuono, M., Colagrossi, a., Colicchio, G., Le Touzé, D., and Graziani, G., “ $\delta$-SPH Model for Simulating Violent Impact Flows," Computer Methods in Applied Mechanics and Engineering, Vol. 200, 2011, pp. 1526-1542.

[44] McBride, E.E., and Fisher, L.J., "Experimental Investigation of the Effect of Rear-Fuselage Shape on Ditching Behavior," NACA TN-2929, 1953.

[45] Streckwall, H., Lindenau, O and Bensch, L., “Aircraft Ditching: a Free Surface/Free Motion Problem," Archives of Civil and Mechanical Engineering, Vol. 7, No. 3, 2007, pp. 177-190. 